\title{
32. HIGH-RESOLUTION ISOTOPIC AND MICROPALEONTOLOGICAL STUDIES OF UPPER PLEISTOCENE SEDIMENTS AT ODP SITE 645, BAFFIN BAY ${ }^{1}$
}

\author{
C. Hillaire-Marcel, ${ }^{2}$ A. DE Vernal, ${ }^{2}$ A. Aksu, ${ }^{3}$ and S. Macko ${ }^{3}$
}

\begin{abstract}
The oxygen and carbon isotopic compositions of the planktonic foraminifer, Neogloboquadrina pachyderma (sinistral), were determined at 20 -cm intervals through the "composite" top $\sim 22 \mathrm{~m}$ of sediments at ODP Site 645 (Holes $645 \mathrm{~B}, 645 \mathrm{C}, 645 \mathrm{~F}$, and $645 \mathrm{G}$ ) and at $10-\mathrm{cm}$ intervals through a 9-m piston core (85-027-016) collected during the Hudson site survey. Quantitative analyses of palynomorphs, notably dinocysts, and of planktonic foraminifers were performed. Organic and nitrogen contents and isotopic composition of nitrogen and carbon in organic matter also were determined. These data provide a high-resolution record of changes that occurred in surface-water masses during the last glacial cycle in Baffin Bay. The basin experienced low planktonic productivity during most of the late Pleistocene, either from dilution in surface water by meltwater discharges from the surrounding ice-sheet or from the presence of a relatively dense sea-ice cover. Peaks of meltwater discharge are indicated by $\delta^{18} \mathrm{O}$ values as low as about $1.5 \%$, correlative $\delta^{13} \mathrm{C}-\delta^{18} \mathrm{O}$ shifts, low concentration of planktonic foraminifers, high concentrations of glacially reworked pre-Quaternary palynomorphs, and low-salinity dinocyst assemblages. As a whole, $\delta^{18} \mathrm{O}$ values ranging between 4.5 and $2.5 \%$ allow the establishment of an ${ }^{18} \mathrm{O}$ stratigraphy spanning isotopic stages 5 to 1 . Because of the poor core recovery, the general paucity of microflora and microfauna, and the possible occurrence of slumping or debris flow at Site 645 , further interpretation remains problematic.
\end{abstract}

\section{INTRODUCTION}

Despite its size ( $200,000 \mathrm{~km}^{2}$ and $2200 \mathrm{~m}$ maximum depth), Baffin Bay constitutes a confined basin with epicontinentaltype sedimentation (Aksu, 1981; Aksu and Piper, 1979, 1987; de Vernal et al., 1987). It is bounded to the north and northwest by the Canadian Arctic Archipelago, to the west by Baffin Island, and to the east by Greenland (Fig. 1). Baffin Bay is connected to the Arctic Ocean through Smith, Jones, and Lancaster sounds, where maximum depths do not exceed $200 \mathrm{~m}$, and to the Labrador Sea through Davis Strait, with $\sim 800$ m maximum depth. During summers, the weak, relatively warm subpolar West Greenland Current flows northward along the west coast of Greenland, penetrating into Baffin Bay to about $72^{\circ} \mathrm{N}$ (Fig. 1). The cold polar Baffin Land Current flows southward along the east coast of Baffin Island and through the Davis Strait. During the winter, most of Baffin Bay is covered by 1 to $3 \mathrm{~m}$ of thick sea ice, while the West Greenland Current is deflected westward around the Davis Strait sill. The Baffin Land Current dominates the surface circulation in Baffin Bay.

Before and during Leg 105, several piston cores were collected by the Hudson, notably during cruises 76-029, 77-027, and $85-027$. The upper $10-20 \mathrm{~cm}$ of the abyssal surface sediments consists predominantly of hemipelagic deposits mixed with varying proportions of ice-rafted debris (Aksu and Piper, 1979). The subsurface deposits largely include ice-rafted sediments, debrites, and turbidites, which indicates a strong influence by glacial erosion from adjacent ice-sheets, glaciomarine transportation, and outwash deposition (Aksu, 1981, 1984; Aksu and Piper, 1987). Two conflicting chronostratigraphies were proposed for Baffin Bay sediments. Using primarily oxygen isotopic data and a few ${ }^{14} \mathrm{C}$ dates for total organic material, Aksu

\footnotetext{
${ }^{1}$ Srivastava, S. P., Arthur, M., Clement, B., et al., 1989. Proc. ODP, Sci. Results, 105: College Station, TX (Ocean Drilling Program).

2 GEOTOP and Sciences de la Terre, Université du Québec à Montréal, B.P. 8888, succ. A, Montréal, Québec H3C 3P8, Canada.

${ }^{3}$ Department of Earth Sciences, Memorial University, St. John's, Newfoundland A1B $3 \times 5$, Canada.
}

(1981, 1983, 1985) suggested slow depositional rates of $\sim 3-5$ $\mathrm{cm} / \mathrm{k}$.y. for the sediments recovered in short piston cores. Recent studies by de Vernal (1986) and de Vernal et al. (1987) used palynological data and accelerator mass spectrometry (AMS) ${ }^{14} \mathrm{C}$ dates for foraminifers to suggest considerably higher sedimentation rates of about $8-10 \mathrm{~cm} / \mathrm{k}$.y. Preliminary paleomagnetic results from Site 645 (Srivastava, Arthur, et al., 1987) indicate the latter chronostratigraphy is correct. High deposition rates in Baffin Bay allow high-resolution studies of paleoclimatic and paleoceanographic changes, especially in relation to the glacial history of the borderlands. From this viewpoint, Site 645 in southwestern Baffin Bay is located strategically for the reconstruction of climatic oscillations and glacial fluctuations in northeastern Canada.

At Site 645 , the uppermost $\sim 22 \mathrm{~m}$ of sediments was cored with some difficulty, and recovery was about $50 \%$. The shipboard lithostratigraphic correlations using sections from five holes $(645 \mathrm{~A}, 645 \mathrm{~B}, 645 \mathrm{C}, 645 \mathrm{~F}$, and $645 \mathrm{G})$ allowed us to construct a composite record with a few gaps (Fig. 2). These gaps and the occurrence of slumping at Site 645 (see the $3.5 \mathrm{kHz}$ profile of Fig. 3) made it difficult to define a clear stratigraphy. Nevertheless, a 9-m piston core (85-027-016) collected nearby, away from the slumping, provided a continuous, albeit brief, sedimentary sequence. The composite sequence of the uppermost $\sim 22 \mathrm{~m}$ of sediments from Site 645 was sampled at approximatly $20-\mathrm{cm}$ intervals to provide a high-resolution paleoceanographic and paleoclimatic record based on (1) $\delta^{18} \mathrm{O}$ and $\delta^{13} \mathrm{C}$ in the dominant planktonic foraminifer, Neogloboquadrina pachyderma (sinistral); (2) organic-carbon and nitrogen concentrations and isotopic compositions; (3) pollen, spore, and dinocyst assemblages; and (4) foraminifer assemblages. The $\delta^{18} \mathrm{O}$ and $\delta^{13} \mathrm{C}$ records from piston Cores 87-027-016 and 76-029-033 (Fig. 1) and palynological results from Core 87-027-16 also are reported.

\section{SAMPLING AND METHODS}

Shipboard lithostratigraphic, colorimetric, and magnetic (susceptibility) correlations among the cores showed that a relatively continuous record can be constructed using overlapping sections from Holes $645 \mathrm{~B}$, $645 \mathrm{C}, 645 \mathrm{~F}$, and $645 \mathrm{G}$ (Fig. 2). Approximately $10 \mathrm{~cm}^{3}$ of sediment was 


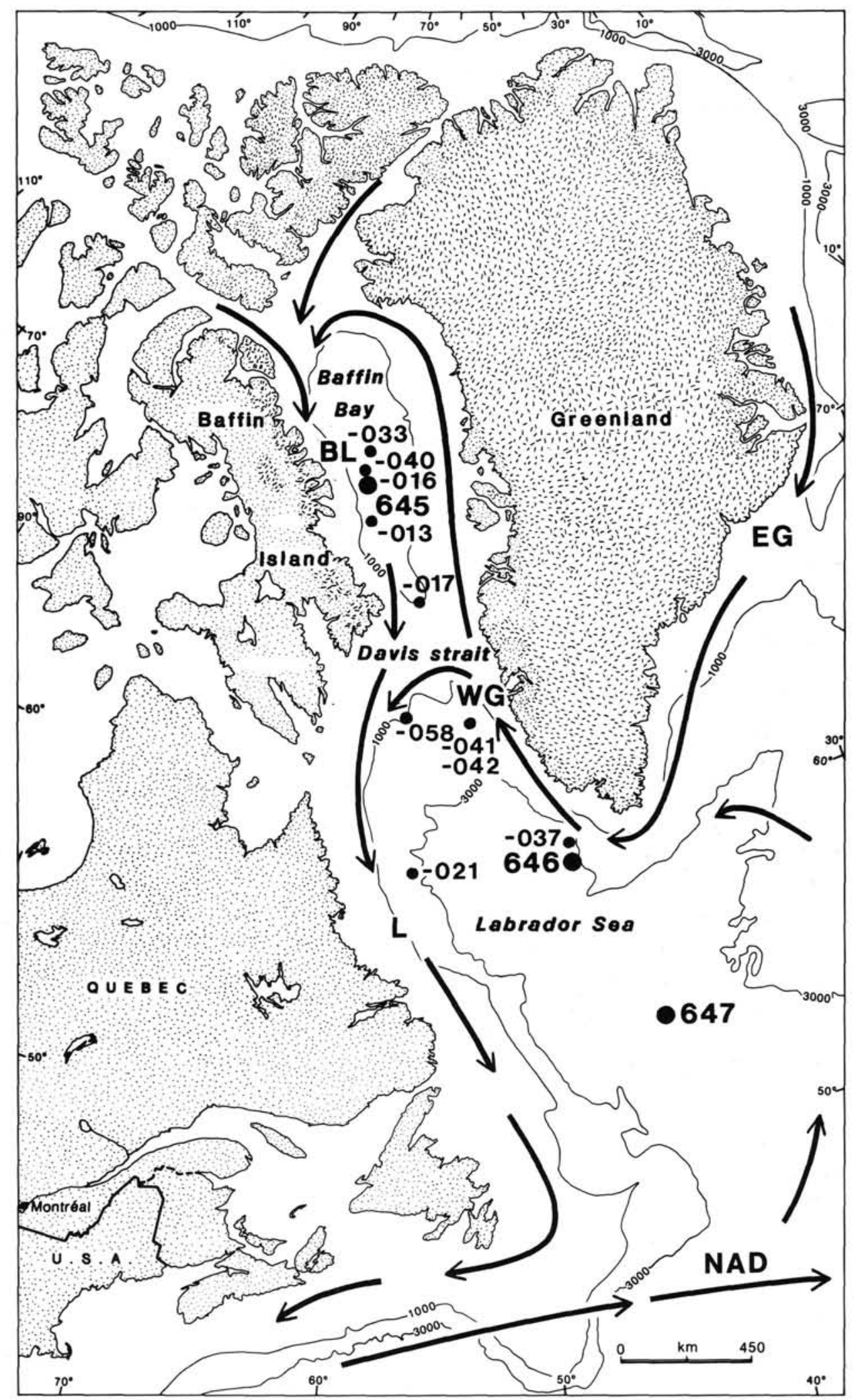

Figure 1. Location map for ODP Sites 645, 646, and 647 and of piston cores mentioned in the text. Modern surface-water circulation is indicated by arrows; $N A D=$ North Atlantic Drift; $E G=$ East Greenland Current; $W G=$ West Greenland Current; $B L=$ Baffin Land Current; $L=$ Labrador Current. 


\section{Site 645}

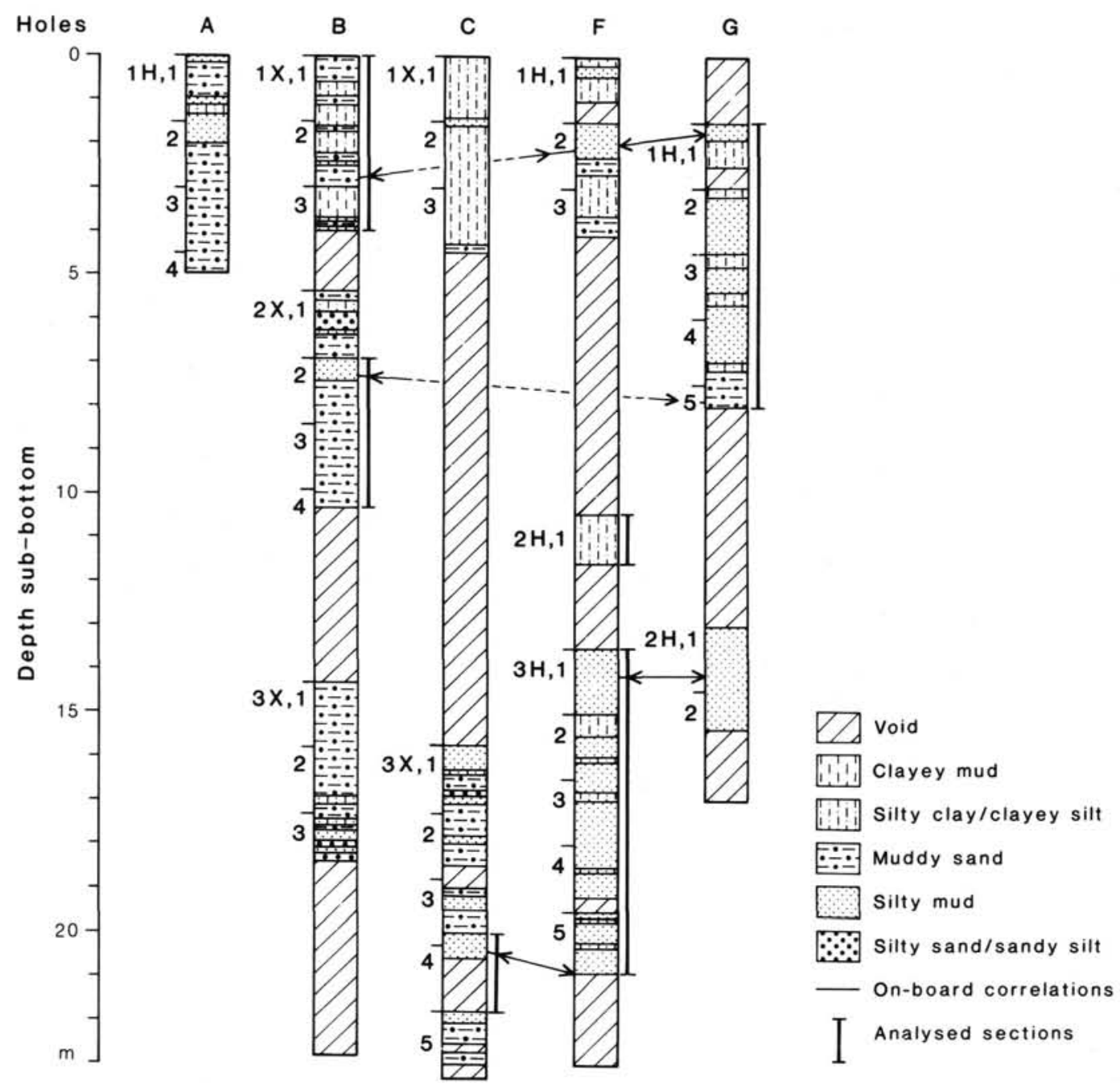

Figure 2. Schematic lithostratigraphy at Site 645 and proposed correlations (according to Srivastava, Arthur, et al., 1987). Solid black bars represent the analyzed sections.

sampled at 20-cm intervals from most core sections in the upper $\sim 22 \mathrm{~m}$ in these holes. Core 85-027-016 was sampled $\left(8 \mathrm{~cm}^{3}\right)$ at 10-cm intervals, which allowed higher-resolution studies.

Foraminifers were prepared for isotopic analysis by sieving the sediments with distilled water through 150 and $250-\mu \mathrm{m}$ screens. Each foraminifer sample was dried at $70^{\circ} \mathrm{C}$ and weighed. The whole 150 - to 250 $\mu \mathrm{m}$ fraction was used to handpick foraminifers. As already mentioned, Neogloboquadrina pachyderma (sinistral) is by far the dominant planktonic foraminifer species. However, this species, is not abundant in all samples (Table 1). $\mathrm{CO}_{2}$ extraction for isotopic analysis was performed according to standard techniques (Duplessy, 1978; Shackleton et al., 1983): reaction at $50^{\circ} \mathrm{C}$ in a water bath, removal of water in a cold trap at $-78^{\circ} \mathrm{C}$, and isotopic measurement in a VG-Isogas Sira 12 . The overall analytical reproducibility was $\pm 0.07 \%$ and $\pm 0.04 \%$ for $\delta^{18} \mathrm{O}$ and $\delta^{13} \mathrm{C}$, respectively (both $\pm 1 \sigma$ values). Analytical tests of aliquots of a well-homogenized batch of $N$. pachyderma yielded reproducible results, with samples containing not more than 10 specimens. Significant $\delta^{18} \mathrm{O}$ values were also obtained from samples with as few as four specimens. However, such results are difficult to interpret because individual isotopic differences and the presence of reworked microfossils may have significantly altered the $\delta^{18} \mathrm{O}$ values. Moreover, fractionation effects may occur during analysis of such small quantities of $\mathrm{CO}_{2}$, and the ana- lytical reproducibility cannot be assessed. All results are reported in Table 1 and referenced to the PDB standard. Only those values obtained from a representative population, $>40$ specimens recovered from each $10-\mathrm{cm}^{3}$ sample, were used in Figure $4\left(4 \mathrm{shells} / \mathrm{cm}^{3}\right.$ represent an influx of approximately one individual $/ \mathrm{cm}^{2} / 20 \mathrm{yr}$ sedimentation).

The few samples containing more than $50 \mathrm{mg}$ of Neogloboquadrina pachyderma were used for AMS ${ }^{14} \mathrm{C}$ measurements. Shells were handpicked after the usual cleaning procedures. Samples were prepared by Beta Analytic without pretreatment because of the small sample size. ${ }^{14} \mathrm{C}$ analyses were performed with the Van de Graaff accelerator at $\mathrm{Zu}$ rich. Results are expressed as conventional ${ }^{14} \mathrm{C}$ dates without isotopic normalization.

Samples $\left(5 \mathrm{~cm}^{3}\right)$ for organic-carbon and nitrogen analyses were stored in a freezer. Stable carbon- and nitrogen-isotope ratios were determined from carbonate-free sediment residues. Lyophylized samples were first acidified with $30 \% \mathrm{HCl}$ and then dried. Next, the weighed samples were combusted in quartz under vacuum in contact with prepurified cupric acid oxide wire and granular copper. The combustion gases were cryogenically purified, and isotopic compositions were measured using a VG 903E mass spectrometer. Organic carbon was measured on a calibrated manometer in the purification line. Total nitrogen content was assessed as the ion intensity of the gas in a calibrated volume of the 


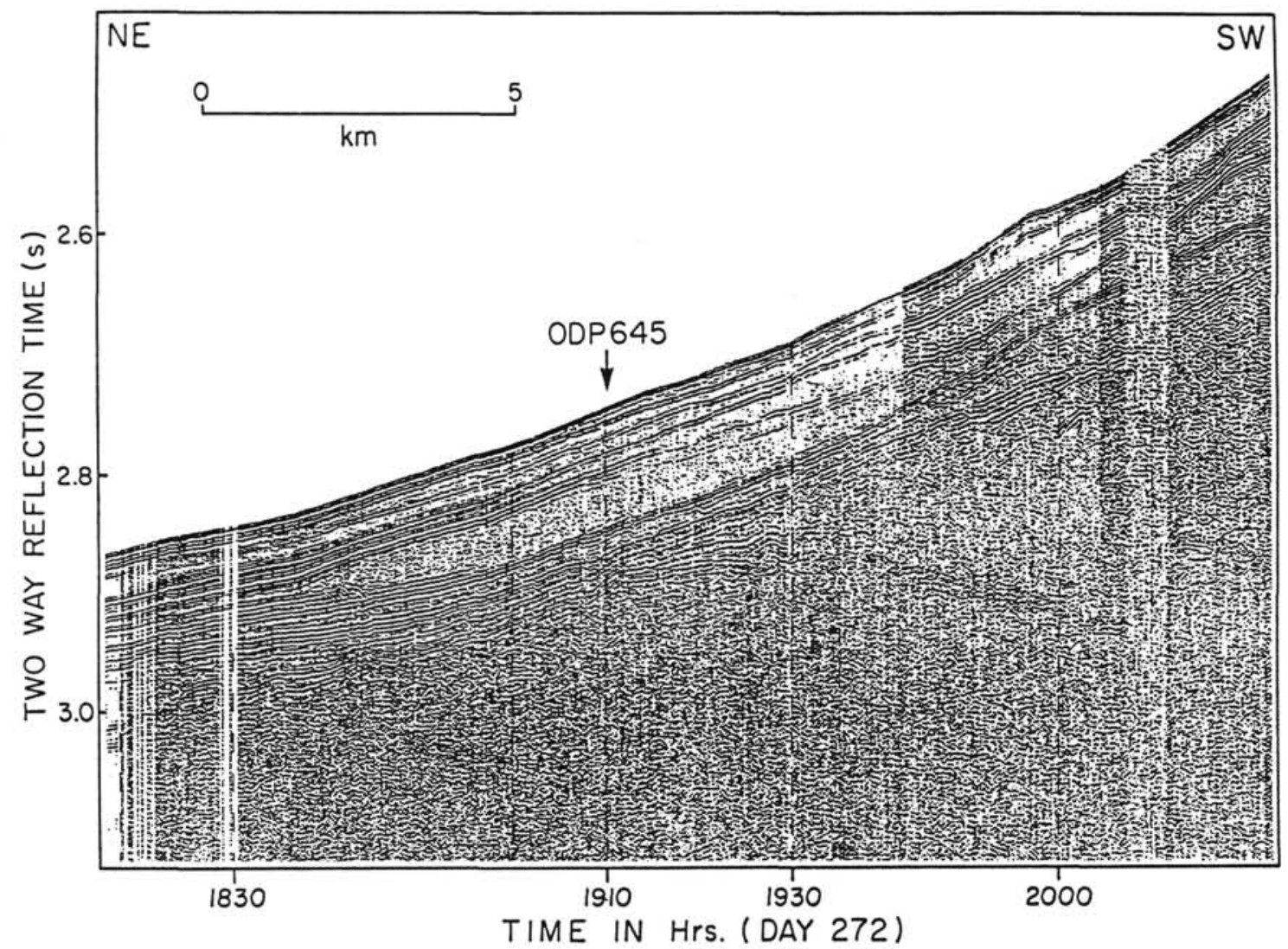

Figure 3. High-resolution seismic $(3.5 \mathrm{kHz})$ profile along Site 645 .

mass spectrometer. Reproducibility is better than $\pm 0.2 \%$. Isotopic compositions are reported relative to $\mathrm{PDB}$ for $\delta^{13} \mathrm{C}$ and to atmospheric nitrogen for $\delta^{15} \mathrm{~N}$ (Table 2).

Samples were prepared for palynological analysis using the technique described by de Vernal and Mudie (this volume). Samples were sieved with distilled water at 10 and $125 \mu \mathrm{m}$ to eliminate fine and coarse particles and then treated with $\mathrm{HCl}(10 \%)$ and $\mathrm{HF}(52 \%)$ to destroy carbonates and to dissolve silicates. Palynomorph concentrations were evaluated on the basis of the marker-grain method (Matthews, 1969). The dinocyst, pollen, spore, and reworked palynomorph concentrations are reported in Table 3. Because of the sparseness of palynoflora, the low counts of Quaternary palynomorphs prevented us from constructing percentage diagrams.

For foraminifer studies, samples were disaggregated in $1 \%$ Calgon solution and wet sieved through a $63-\mu \mathrm{m}$ screen. The coarse fractions were dried in an oven, then weighed. The fine fractions were saved for further studies. Planktonic foraminifers were separated at the $63-\mu \mathrm{m}$ fraction, identified, and then counted, following the method described by Aksu (1985). Total foraminifer abundances were converted to "specimens per gram dry weight sediment." Individual species abundances were displayed as percentages of the total planktonic foraminifers.

\section{OXYGEN-ISOTOPE RECORD}

Although large-amplitude shifts (from 0.3 to $4.6 \%$ ) were observed throughout the $\delta^{18} \mathrm{O}$ record, most values range from 2.5 to $4.6 \%$. The lower values were recorded in samples essentially containing very few foraminifers (Table 1). Today, the upper water layer (from 0 to $250 \mathrm{~m}$ deep) has temperatures ranging from 1 to $+1.8^{\circ} \mathrm{C}$ (Muench, 1971). It would be difficult to hypothesize higher temperatures at this latitude during the last glaciation. Hence, most of the ${ }^{18} \mathrm{O}$ changes in planktonic foraminifers must be attributed to changes in the isotopic composition of the surface-water masses. Assuming an isotopic composition range of approximately $-30 /-20 \%$ vs. standard mean ocean water
(SMOW) for meltwater from the surrounding ice caps during the last glaciation (Dansgaard et al., 1971; Broecker, 1975), surface-water salinity ranging from approximately 31 to $35 \%$ can be extrapolated from today's conditions in Baffin Bay (Tan and Strain, 1980; Bédard et al., 1981). Rapid mixing and/or evacuation of the ice meltwater seems probable in view of the brevity of the "18 $\mathrm{O}$-depleted" episodes.

The short meltwater peaks of the $\delta^{18} \mathrm{O}$ record can be screened out by retaining only samples containing at least 40 tests (i.e., about 4 specimens $/ \mathrm{cm}^{3}$ ). The filtered records (Figs. 4, 5, 6, and 7) are similar to those obtained from isotopic measurements on the same species in piston cores from the Labrador Sea. The ${ }^{18} \mathrm{O}$ stratigraphy of the northeast Labrador Sea, on the incoming West Greenland Current trajectory (Core 75-041/042: Fillon and Duplessy, 1980) and that of the northwest Labrador Sea, on the outgoing Labrador Current path (Core 75-058: Fillon, 1985; Core 84-030-021: de Vernal and Hillaire-Marcel, 1987), both show $\delta^{18} \mathrm{O}$ ranges of about $2.5 \%$, between $+4.7 \%$ (isotopic stage 2: Cores 75-041 and 74-042) and $+2.1 \%$ (isotopic stage 1 : Cores 84-030 through 84-021). On the basis of the highest $\delta^{18} \mathrm{O}$ values $(>+4 \%$ ) of isotopic stages 2 and 4 , a good correlation can be seen among Cores 105-645G-1H, 85-027-016, and 76029-033 (Fig. 8), which were all collected in the western part of Baffin Bay, and with Cores 77-027-017 (Mudie and Aksu, 1984) and 75-041 and 75-042 (Fillon and Duplessy, 1980), from Davis Strait and the northern Labrador Sea, respectively. Despite the isotopic signal of local meltwater discharge events, a relatively clear isotope stratigraphy can be established in Baffin Bay.

The gravity and piston cores from the Hudson expeditions and Core 105-645B-1X all show a short Holocene record. The $\delta^{18} \mathrm{O}$ values lower than $3 \%$ appear only at or above $50 \mathrm{~cm}$ subbottom in just a few cores. An AMS ${ }^{14} \mathrm{C}$ date of $10,590 \pm 130 \mathrm{yr}$ 
Table 1. Oxygen- and carbon-isotope compositions in Neogloboquadrina pachyderma (sinistral) from Baffin Bay deep-sea cores. *

\begin{tabular}{|c|c|c|c|c|c|c|c|c|c|c|c|}
\hline $\begin{array}{l}\text { Laboratory } \\
\text { number }\end{array}$ & Core/section & $\begin{array}{l}\text { Depth } \\
(\mathrm{cm})\end{array}$ & $\begin{array}{l}\text { Number } \\
\text { of shells } \\
\text { analyzed }\end{array}$ & $\begin{array}{c}\delta^{13} \mathrm{C} \\
(\mathrm{PDB})\end{array}$ & $\begin{array}{l}\delta^{18} \mathrm{O} \\
\text { (PDB) }\end{array}$ & $\begin{array}{l}\text { Laboratory } \\
\text { number }\end{array}$ & Core/section & $\begin{array}{l}\text { Depth } \\
(\mathrm{cm})\end{array}$ & $\begin{array}{l}\text { Number } \\
\text { of shells } \\
\text { analyzed }\end{array}$ & $\begin{array}{c}\delta^{13} \mathrm{C} \\
(\mathrm{PDB})\end{array}$ & $\begin{array}{c}\delta^{18} \mathrm{O} \\
\text { (PDB) }\end{array}$ \\
\hline UQ-C54-02 & $105-645 \mathrm{~B}-1 \mathrm{X}-1$ & $78-80$ & 100 & -0.15 & +2.32 & UQ-C54-47 & $645 \mathrm{~F}-3 \mathrm{H}-3$ & $48-50$ & 100 & -0.61 & +3.58 \\
\hline UQ-C54-01 & $645 \mathrm{~B}-1 \mathrm{X}-1$ & $115-117$ & 46 & -0.10 & +3.10 & UQ-C54-48 & $645 \mathrm{~F}-3 \mathrm{H}-3$ & $68-70$ & 100 & -0.83 & +3.14 \\
\hline UQ-C54-03 & $645 \mathrm{~B}-1 \mathrm{X}-2$ & $58-60$ & 43 & -0.10 & +3.10 & UQ-C54-49 & $645 \mathrm{~F}-3 \mathrm{H}-3$ & 87-89 & 3 & - & - \\
\hline UQ-C54-04 & $645 B-1 X-2$ & $78-80$ & 14 & -1.02 & +2.50 & UQ-C54-50 & $645 \mathrm{~F}-3 \mathrm{H}-3$ & $128-130$ & 3 & - & - \\
\hline UQ-C54-05 & $645 B-1 X-2$ & $98-100$ & 100 & -0.33 & +2.71 & & & & & & \\
\hline UQ-C54-06 & $645 B-1 X-2$ & $117-119$ & 100 & -0.01 & +3.36 & UQ-C54-51 & $645 \mathrm{~F}-3 \mathrm{H}-4$ & $50-52$ & 91 & -0.81 & +3.32 \\
\hline UQ-C54-07 & $645 \mathrm{~B}-1 \mathrm{X}-2$ & $136-138$ & 100 & +0.12 & +4.46 & UQ-C 54-52 & $645 \mathrm{~F}-3 \mathrm{H}-4$ & $87-89$ & 25 & -0.29 & $\begin{array}{l}+4.03 \\
+4.59\end{array}$ \\
\hline UQ-C $54-60$ & $645 \mathrm{G}-1 \mathrm{H}-1$ & 07-09 & 100 & +0.55 & +3.81 & UQ-C54-53 & $645 \mathrm{~F}-3 \mathrm{H}-4$ & $112-114$ & 25 & -0.36 & +4.59 \\
\hline UQ-C54-61 & $645 \mathrm{G}-1 \mathrm{H}-1$ & $26-28$ & 31 & -1.31 & +0.71 & UQ-C54-54 & $645 \mathrm{~F}-3 \mathrm{H}-5$ & $08-10$ & 92 & -0.64 & +4.07 \\
\hline UQ-C54-62 & $645 \mathrm{G}-1 \mathrm{H}-1$ & $47-49$ & 100 & +0.03 & +3.45 & UQ-C54-55 & $645 \mathrm{~F}-3 \mathrm{H}-5$ & $28-30$ & 28 & -0.77 & +3.67 \\
\hline UQ-C54-63 & $645 \mathrm{G}-1 \mathrm{H}-1$ & $67-69$ & 100 & +0.12 & +4.11 & UQ-C54-56 & $645 \mathrm{~F}-3 \mathrm{H}-5$ & $48-50$ & 15 & -0.69 & +3.57 \\
\hline UQ-C54-64 & $645 \mathrm{G}-1 \mathrm{H}-1$ & $91-93$ & 100 & +0.13 & +3.99 & UQ-C54-57 & $645 \mathrm{~F}-3 \mathrm{H}-5$ & $67-69$ & 16 & -0.30 & +4.08 \\
\hline UQ-C54-65 & $645 \mathrm{G}-1 \mathrm{H}-2$ & $01-03$ & 100 & -0.10 & +3.64 & UQ-C54-58 & $645 \mathrm{~F}-3 \mathrm{H}-5$ & $107-109$ & 10 & -1.02 & +4.01 \\
\hline UQ-C54-66 & $645 \mathrm{G}-1 \mathrm{H}-2$ & $18-20$ & 100 & +0.44 & +4.52 & UQ-C54-59 & $645 \mathrm{~F}-3 \mathrm{H}-5$ & $128-130$ & 100 & -0.47 & +4.25 \\
\hline UQ-C54-67 & $645 \mathrm{G}-1 \mathrm{H}-2$ & $38-40$ & 100 & +0.46 & +4.62 & & & & & & \\
\hline UQ-C54-68 & $645 \mathrm{G}-1 \mathrm{H}-2$ & $57-59$ & 100 & +0.39 & +4.27 & UQ-C54-23 & $645 \mathrm{C}-3 \mathrm{H}-4$ & $19-21$ & 100 & -0.72 & +4.17 \\
\hline UQ-C54-69 & $645 \mathrm{G}-1 \mathrm{H}-2$ & $78-80$ & 100 & +0.33 & +4.01 & UQ-C54-24 & $645 \mathrm{C}-3 \mathrm{H}-4$ & $36-39$ & 100 & -0.68 & +3.68 \\
\hline UQ-C54-70 & $645 \mathrm{G}-1 \mathrm{H}-2$ & $97-99$ & 100 & +0.60 & +4.17 & UQ-C54-25 & $645 \mathrm{C}-3 \mathrm{H}-5$ & $08-10$ & 100 & -0.81 & +3.51 \\
\hline UQ-C 54-71 & $645 \mathrm{G}-1 \mathrm{H}-2$ & $117-119$ & 100 & +0.30 & +3.77 & UQ-C54-26 & $645 \mathrm{C}-3 \mathrm{H}-5$ & $48-50$ & 100 & -0.24 & +3.27 \\
\hline UQ-C54-72 & $645 \mathrm{G}-1 \mathrm{H}-2$ & $136-138$ & 63 & -0.01 & +3.80 & UQ-C54-27 & $645 \mathrm{C}-3 \mathrm{H}-5$ & $92-94$ & 79 & -0.02 & +2.91 \\
\hline UQ-C $54-73$ & $645 \mathrm{G}-1 \mathrm{H}-3$ & $01-03$ & 42 & -0.08 & +3.08 & & & & & & \\
\hline UQ-C54-74 & $645 \mathrm{G}-1 \mathrm{H}-3$ & $37-39$ & 100 & +0.11 & +3.71 & UQ-D45-86 & 85-027-016TWC & 5 & 0 & - & - \\
\hline UQ-C54-75 & $645 \mathrm{G}-1 \mathrm{H}-3$ & $57-59$ & 100 & +0.20 & +3.91 & UQ-D45-87 & & 14 & 0 & - & - \\
\hline UQ-C $54-76$ & $645 \mathrm{G}-1 \mathrm{H}-3$ & $76-79$ & 100 & +0.18 & +2.90 & UQ-D45-88 & & 20 & 36 & +0.13 & +1.74 \\
\hline UQ-C $54-39$ & $645 \mathrm{G}-1 \mathrm{H}-3$ & $97-99$ & 100 & -0.13 & +2.87 & UQ-D45-89 & & 31 & 7 & - & +1.24 \\
\hline UQ-C $54-77$ & $645 \mathrm{G}-1 \mathrm{H}-3$ & $118-120$ & 38 & -1.31 & +1.10 & UQ-D45-90 & & 40 & 18 & -0.07 & +1.97 \\
\hline UQ-C54-78 & $645 \mathrm{G}-1 \mathrm{H}-4$ & $08-10$ & 100 & +0.42 & +3.40 & UQ-D45-91 & & 50 & 32 & +0.29 & +2.90 \\
\hline UQ-C54-79 & $645 \mathrm{G}-1 \mathrm{H}-4$ & $26-28$ & 55 & +0.20 & +2.74 & UQ-D45-92 & & 61 & 100 & +0.06 & +3.41 \\
\hline UQ-C54-80 & $645 \mathrm{G}-1 \mathrm{H}-4$ & $46-48$ & 35 & -0.83 & +0.69 & UQ-D45-93 & & 71 & 27 & +0.05 & +3.05 \\
\hline UQ-C54-81 & $645 \mathrm{G}-1 \mathrm{H}-4$ & $67-69$ & 100 & +0.33 & +2.68 & UQ-D45-94 & & 81 & 54 & -0.03 & +3.09 \\
\hline UQ-C54-82 & $645 \mathrm{G}-1 \mathrm{H}-4$ & $87-89$ & 66 & +0.04 & +2.36 & UQ-D45-95 & & 90 & 15 & -0.51 & +2.69 \\
\hline UQ-C54-83 & $645 \mathrm{G}-1 \mathrm{H}-4$ & $107-109$ & 36 & -1.14 & +0.33 & UQ-D45-96 & & 101 & 2 & - & - \\
\hline UQ-C $54-84$ & $645 \mathrm{G}-1 \mathrm{H}-5$ & $01-03$ & 7 & +0.25 & +2.65 & UQ-D45-97 & & 112 & 88 & +0.04 & +3.52 \\
\hline UQ-C54-85 & $645 \mathrm{G}-1 \mathrm{H}-5$ & $18-20$ & 100 & +0.55 & +4.18 & UQ-D45-98 & & 120 & 1 & - & - \\
\hline UQ-C54-86 & $645 \mathrm{G}-1 \mathrm{H}-5$ & $56-58$ & 100 & +0.56 & +3.17 & UQ-D45-99 & & 130 & 100 & +0.04 & +3.09 \\
\hline UQ-C54-87 & $645 \mathrm{G}-1 \mathrm{H}-5$ & $77-79$ & 100 & -0.08 & +3.97 & UQ-D45-100 & & 141 & 14 & - & +3.67 \\
\hline UQ-C54-88 & $645 \mathrm{G}-1 \mathrm{H}-5$ & $98-100$ & 100 & -0.35 & +4.09 & UQ-D45-101 & & 150 & 4 & -0.25 & +2.70 \\
\hline UQ-C54-89 & $645 \mathrm{G}-1 \mathrm{H}-5$ & $118-120$ & 100 & -0.35 & +3.91 & & & & & & \\
\hline \multirow[t]{2}{*}{ UQ-C $54-90$} & $645 \mathrm{G}-1 \mathrm{H}-5$ & $138-140$ & 29 & -0.47 & +4.02 & UQ-D45-1 & $85-027-016-\mathrm{P}$ & 1 & 14 & -0.52 & +2.27 \\
\hline & & & & & & UQ-D45-2 & & 10 & 56 & +0.28 & +2.26 \\
\hline UQ-C54-08 & $645 \mathrm{~B}, 2 \mathrm{X}-2$ & $28-30$ & 100 & -0.32 & +4.21 & UQ-D45-3 & & 21 & 8 & -0.97 & +2.51 \\
\hline UQ-C54-09 & $645 \mathrm{~B}-2 \mathrm{X}-2$ & $58-60$ & 95 & -0.23 & +3.52 & UQ-D45-4 & & 31 & 51 & +0.07 & +3.20 \\
\hline UQ-C54-10 & $645 \mathrm{~B}-2 \mathrm{X}-2$ & $78-80$ & 100 & -0.29 & +3.31 & UQ-D45-5 & & 44 & 100 & +0.15 & +3.03 \\
\hline UQ-C54-11 & $645 \mathrm{~B}-2 \mathrm{X}-2$ & $94-96$ & 100 & -0.30 & +2.65 & UQ-D45-6 & & 51 & 100 & +0.07 & +3.07 \\
\hline UQ-C54-12 & $645 B-2 X-2$ & $117-119$ & 100 & -0.26 & +3.61 & UQ-D45-7 & & 60 & 100 & +0.08 & +3.16 \\
\hline UQ-C54-13 & $645 \mathrm{~B}-2 \mathrm{X}-2$ & $138-140$ & 100 & -0.25 & +3.74 & UQ-D45-8 & & 71 & 100 & -0.31 & +2.46 \\
\hline UQ-C54-14 & $645 \mathrm{~B}-2 \mathrm{X}-3$ & $01-03$ & 100 & -0.25 & +3.46 & UQ-D45-9 & & 80 & 35 & +0.04 & +3.19 \\
\hline UQ-C54-15 & $645 \mathrm{~B}-2 \mathrm{X}-3$ & $18-20$ & 100 & -0.57 & +3.05 & UQ-D45-10 & & 99 & 100 & -0.29 & +2.47 \\
\hline UQ-C54-16 & $645 B-2 X-3$ & $38-40$ & 100 & -0.21 & +3.45 & UQ-D45-11 & & 110 & 56 & -0.85 & +2.60 \\
\hline UQ-C54-17 & $534 \mathrm{~B}-2 \mathrm{X}-3$ & $59-60$ & 100 & -0.31 & +3.69 & UQ-D45-12 & & 120 & 2 & - & - \\
\hline UQ-C54-18 & $645 \mathrm{~B}-2 \mathrm{X}-3$ & $77-79$ & 100 & -0.30 & +3.79 & UQ-D45-13 & & 131 & 0 & - & - \\
\hline UQ-C $54-19$ & $645 B-2 X-3$ & $97-99$ & 100 & -0.29 & +3.66 & UQ-D45-14 & & 140 & 28 & -1.17 & +2.59 \\
\hline UQ-C54-20 & $645 B-2 X-3$ & $115-117$ & 100 & -0.49 & +3.46 & UQ-D45-15 & & 151 & 73 & -0.50 & +3.04 \\
\hline UQ-C $54-21$ & $645 B-2 X-4$ & $18-20$ & 100 & -0.39 & +3.71 & UQ-D45-16 & & 160 & 0 & - & - \\
\hline \multirow[t]{2}{*}{ UQ-C $54-22$} & $645 B-2 X-4$ & $38-40$ & 100 & -0.36 & +3.56 & UQ-D45-17 & & 171 & 38 & -0.30 & +3.81 \\
\hline & & & & & & UQ-D45-18 & & 180 & 1 & - & - \\
\hline UQ-C54-28 & $645 \mathrm{~F}-2 \mathrm{H}-1$ & 07-09 & 100 & -0.03 & +1.39 & UQ-D45-19 & & 191 & 0 & - & - \\
\hline UQ-C54-29 & $645 \mathrm{~F}-2 \mathrm{H}-1$ & $28-30$ & 100 & -0.04 & +3.49 & UQ-D45-20 & & 200 & 0 & - & - \\
\hline UQ-C54-30 & $645 \mathrm{~F}-2 \mathrm{H}-1$ & $50-52$ & 100 & +0.06 & +3.41 & UQ-D45-21 & & 210 & 0 & - & - \\
\hline UQ-C54-31 & $645 \mathrm{~F}-2 \mathrm{H}-1$ & $88-90$ & 8 & -0.26 & +1.47 & UQ-D45-22 & & 221 & 0 & - & - \\
\hline & & & & & & UQ-D45-23 & & 230 & 0 & - & - \\
\hline UQ-C54-32 & $645 \mathrm{~F}-3 \mathrm{H}-1$ & $02-04$ & 100 & -0.22 & +4.21 & UQ-D45-24 & & 241 & 0 & - & - \\
\hline UQ-C54-33 & $645 \mathrm{~F}-3 \mathrm{H}-1$ & $19-21$ & 100 & -0.29 & +3.95 & UQ-D45-25 & & 253 & 0 & - & - \\
\hline UQ-C54-34 & $645 \mathrm{~F}-3 \mathrm{H}-1$ & $37-39$ & 100 & -0.41 & +3.55 & UQ-D45-26 & & 262 & 30 & $-1 . i 0$ & +3.54 \\
\hline UQ-C54-35 & $645 \mathrm{~F}-3 \mathrm{H}-1$ & $57-59$ & 100 & -0.19 & +4.07 & UQ-D45-27 & & 271 & 100 & -0.31 & +2.73 \\
\hline UQ-C54-36 & $645 \mathrm{~F}-3 \mathrm{H}-1$ & $77-79$ & 100 & -0.37 & +3.99 & UQ-D45-28 & & 277 & 100 & -0.07 & +4.01 \\
\hline UQ-C54-37 & $645 \mathrm{~F}-3 \mathrm{H}-1$ & $98-100$ & 100 & -0.27 & +4.00 & UQ-D45-29 & & 288 & 8 & -0.40 & +3.51 \\
\hline UQ-C54-38 & $645 \mathrm{~F}-3 \mathrm{H}-1$ & $117-119$ & 51 & -0.84 & +3.15 & UQ-D45-30 & & 299 & 11 & +0.09 & +3.02 \\
\hline UQ-C54-40 & $645 \mathrm{~F}-3 \mathrm{H}-1$ & $140-142$ & 100 & -0.83 & +3.36 & UQ-D45-31 & & 310 & 12 & -0.10 & +3.34 \\
\hline UQ-C54-4I & $645 \mathrm{~F}-3 \mathrm{H}-2$ & $08-10$ & 100 & +0.44 & +3.54 & UQ-D45-32 & & 319 & 0 & - & - \\
\hline UQ-C $54-42$ & $645 \mathrm{~F}-3 \mathrm{H}-2$ & $28-30$ & 36 & -0.82 & +0.58 & UQ-D45-33 & & 330 & 100 & -0.08 & +2.70 \\
\hline UQ-C 54.43 & $645 \mathrm{~F}-3 \mathrm{H}-2$ & $108-110$ & 100 & -0.48 & +3.36 & UQ-D45-34 & & 341 & 0 & - & - \\
\hline UQ-C54-44 & $645 \mathrm{~F}-3 \mathrm{H}-2$ & $144-146$ & 66 & -0.20 & +4.52 & UQ-D45-35 & & 350 & 3 & - & - \\
\hline & & & & & & UQ-D45-36 & & 360 & 100 & -0.71 & +4.09 \\
\hline UQ-C $54-45$ & $645 \mathrm{~F}-3 \mathrm{H}-3$ & $07-09$ & 25 & -1.48 & +1.87 & UQ-D45-37 & & 371 & 100 & +0.16 & +4.09 \\
\hline UQ-C54-46 & $645 \mathrm{~F}-3 \mathrm{H}-3$ & $31-33$ & 25 & -0.40 & +4.54 & UQ-D45-38 & & 395 & 2 & - & - \\
\hline & & & & & & UQ-D45-39 & & 405 & 9 & - & - \\
\hline
\end{tabular}

Table 1 (continued). 
Table 1 (continued).

\begin{tabular}{|c|c|c|c|c|c|}
\hline $\begin{array}{l}\text { Laboratory } \\
\text { number }\end{array}$ & Core/section & $\begin{array}{l}\text { Depth } \\
(\mathrm{cm})\end{array}$ & $\begin{array}{l}\text { Number } \\
\text { of shells } \\
\text { analyzed }\end{array}$ & $\begin{array}{c}\delta^{13} \mathrm{C} \\
\text { (PDB) }\end{array}$ & $\begin{array}{c}\delta^{18} \mathrm{O} \\
\text { (PDB) }\end{array}$ \\
\hline UQ-D45-40 & & 416 & 4 & -0.32 & +1.32 \\
\hline UQ-D45-41 & & 426 & 100 & +0.05 & +3.74 \\
\hline UQ-D45-42 & & 436 & 0 & - & - \\
\hline UQ-D45-43 & & 446 & 0 & - & - \\
\hline UQ-D45-44 & & 455 & 0 & - & - \\
\hline UQ-D45-45 & & 465 & 100 & +0.28 & +2.57 \\
\hline UQ-D45-46 & & 474 & 100 & +0.57 & +2.84 \\
\hline UQ-D45-47 & & 485 & 100 & +0.35 & +2.95 \\
\hline UQ-D45-48 & & 494 & 1 & - & - \\
\hline UQ-D45-49 & & 505 & 0 & - & - \\
\hline UQ-D45-50 & & 514 & 2 & - & - \\
\hline UQ-D45-51 & & 525 & 100 & +0.13 & +2.77 \\
\hline UQ-D45-52 & & 534 & 16 & -0.23 & +2.82 \\
\hline UQ-D45-53 & & 545 & 2 & - & - \\
\hline UQ-D45-54 & & 554 & 1 & - & - \\
\hline UQ-D45-55 & & 565 & 9 & - & - \\
\hline UQ-D45-56 & & 574 & 0 & - & - \\
\hline UQ-D45-57 & & 585 & 1 & - & - \\
\hline UQ-D45-58 & & 594 & 6 & - & - \\
\hline UQ-D45-59 & & 606 & 0 & - & - \\
\hline UQ-D45-60 & & 614 & 0 & - & - \\
\hline UQ-D45-61 & & 625 & 120 & +0.55 & +2.63 \\
\hline UQ-D45-62 & & 634 & 95 & +0.24 & +3.17 \\
\hline UQ-D45-63 & & 645 & 100 & +0.26 & +3.07 \\
\hline UQ-D45-64 & & 654 & 0 & - & - \\
\hline UQ-D45-65 & & 665 & 100 & +0.18 & +1.54 \\
\hline UQ-D45-66 & & 674 & 100 & +0.01 & +1.69 \\
\hline UQ-D45-67 & & 685 & 14 & -0.60 & +2.29 \\
\hline UQ-D45-68 & & 694 & 4 & -0.01 & +1.52 \\
\hline UQ-D45-69 & & 705 & 100 & -0.42 & +1.57 \\
\hline UQ-D45-70 & & 714 & 20 & -0.34 & +1.69 \\
\hline UQ-D45-71 & & 725 & 21 & -0.43 & +4.16 \\
\hline UQ-D45-72 & & 736 & 100 & -0.31 & +3.88 \\
\hline UQ-D45-73 & & 745 & 0 & - & - \\
\hline UQ-D45-74 & & 754 & 100 & -0.37 & +3.71 \\
\hline UQ-D45-75 & & 765 & 0 & - & - \\
\hline UQ-D45-76 & & 774 & 100 & -0.25 & +4.14 \\
\hline UQ-D45-77 & & 785 & 100 & +0.13 & +4.18 \\
\hline UQ-D45-78 & & 794 & 100 & -0.11 & +3.29 \\
\hline UQ-D45-79 & & 805 & 100 & -0.45 & +2.56 \\
\hline UQ-D45-80 & & 815 & 0 & - & - \\
\hline UQ-D45-81 & & 824 & 100 & +0.00 & +2.51 \\
\hline UQ-D45-82 & & 835 & 0 & - & - \\
\hline UQ-D45-83 & & 845 & 12 & +0.35 & +2.04 \\
\hline UQ-D45-84 & & 857 & 0 & - & - \\
\hline UQ-D45-85 & & 876 & 0 & - & - \\
\hline UQ-D63-1 & 76-029-033-P & $10-12$ & 46 & +0.30 & +1.62 \\
\hline UQ-D63-2 & & $15-17$ & 10 & - & - \\
\hline UQ-D63-3 & & $30-32$ & 30 & -0.15 & +2.77 \\
\hline UQ-D63-4 & & $35-37$ & 50 & -0.09 & +3.30 \\
\hline UQ-D63-5 & & $40-42$ & 30 & -0.20 & +4.12 \\
\hline UQ-D63-6 & & $45-47$ & 120 & -0.47 & +2.69 \\
\hline UQ-D63-7 & & $70-72$ & 95 & -0.06 & +3.82 \\
\hline UQ-D63-8a & & $85-87$ & 60 & +0.01 & +3.41 \\
\hline UQ-D63-8b & & $85-87$ & 100 & -0.22 & +3.01 \\
\hline UQ-D63-9 & & $90-92$ & 100 & -0.06 & +4.23 \\
\hline UQ-D63-10 & & 99-101 & 100 & -0.05 & +4.04 \\
\hline UQ-D63-11 & & $155-157$ & 70 & -0.12 & +3.80 \\
\hline UQ-D63-12 & & $165-167$ & 30 & -0.55 & +4.31 \\
\hline UQ-D63-13 & & $250-253$ & 100 & +0.11 & +4.24 \\
\hline UQ-D63-14 & & $270-273$ & 100 & -0.45 & +3.92 \\
\hline UQ-D63-15 & & $290-293$ & 100 & -0.29 & +3.99 \\
\hline UQ-D63-16 & & $299-303$ & 100 & -0.13 & +3.61 \\
\hline UQ-D63-17 & & $309-313$ & 100 & -0.13 & +4.24 \\
\hline UQ-D63-18 & & $320-322$ & 100 & +0.02 & +3.62 \\
\hline UQ-D63-19 & & $330-333$ & 30 & -0.33 & +3.37 \\
\hline UQ-D63-20 & & $340-343$ & 100 & +0.25 & +4.12 \\
\hline UQ-D63-21 & & $350-353$ & 16 & -0.20 & +3.58 \\
\hline UQ-D63-22 & & $370-373$ & 100 & +0.39 & +3.03 \\
\hline UQ-D63-23 & & $380-383$ & 100 & +0.17 & +2.30 \\
\hline UQ-D63-24 & & $440-443$ & 65 & +0.36 & +2.65 \\
\hline UQ-D63-25 & & $470-473$ & 6 & -0.13 & +2.77 \\
\hline UQ-D63-26 & & $490-493$ & 100 & +0.02 & +3.16 \\
\hline
\end{tabular}

*When possible, 100 tests were handpicked for isotopic measurement. Smaller numbers of specimens represent the maximum number available in the total sample $\left(10 \mathrm{~cm}^{3} /\right.$ Site 645 and Core $76-029-033 ; 8 \mathrm{~cm}^{3} /$ Core $76-029$. 016).

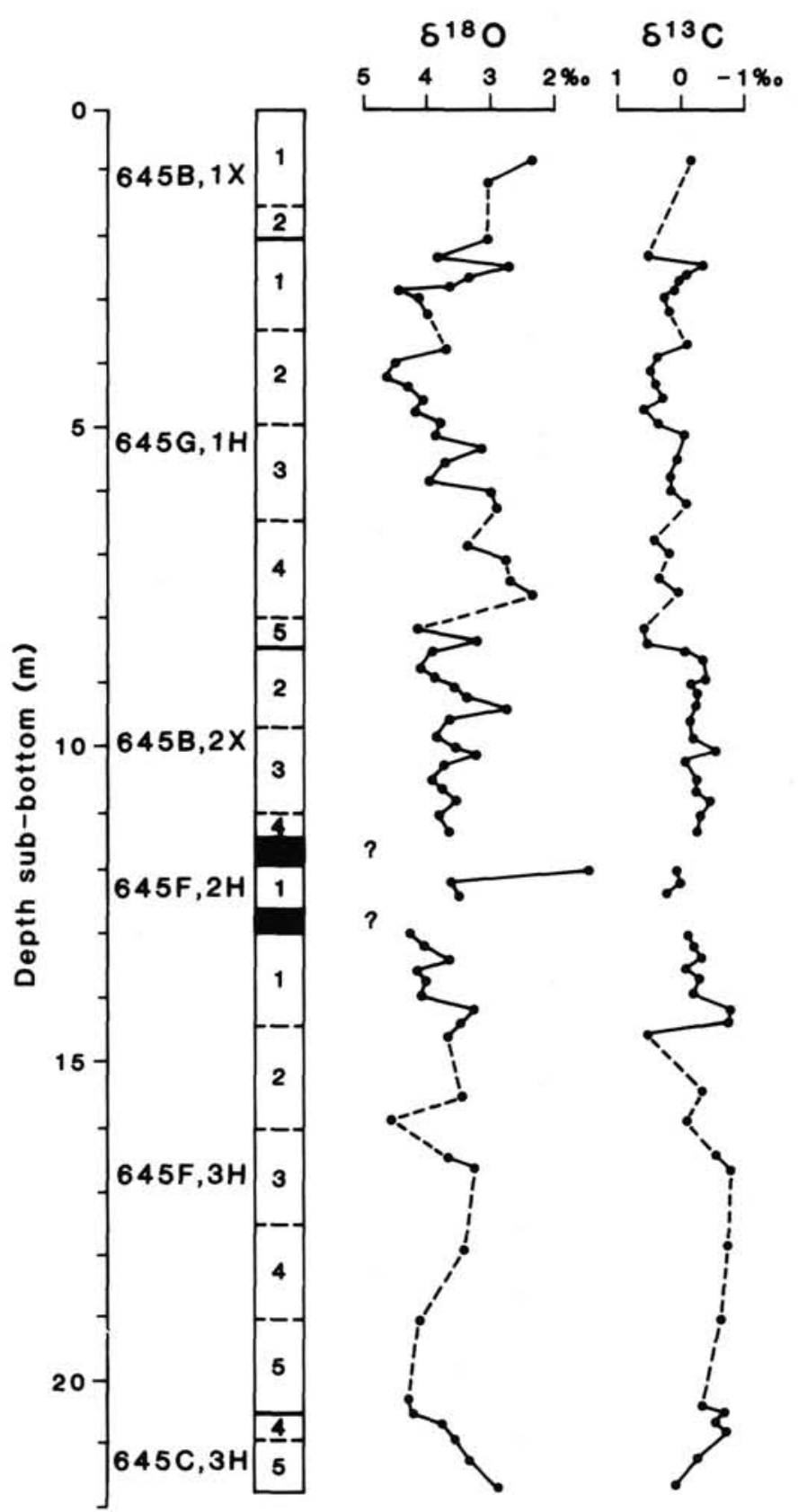

Figure 4. "Screened" $\delta^{13} \mathrm{C}$ and $\delta^{18} \mathrm{O}$ profiles in the composite sequence of Site 645 (Holes 645B and 645C: $70^{\circ} 27^{\prime} 43 \mathrm{~N}, 64^{\circ} 39^{\prime} 26 \mathrm{~W}$; water depth $=2011 \mathrm{~m}$. Holes $645 \mathrm{~F}$ and $645 \mathrm{G}: 70^{\circ} 27^{\prime} 48 \mathrm{~N}, 64^{\circ} 39^{\prime} 29 \mathrm{~W}$; water depth $=2016 \mathrm{~m}$ ). All data points from small populations (i.e., $<40$ individuals $/ 10 \mathrm{~cm}^{3}$ ) have been excluded. Dotted lines join data points over sample gaps.

b.p. indicates that the isotopic stage 2 /stage 1 transition is recorded at about $50 \mathrm{~cm}$ sub-bottom in Core 85-027-016TWC (Scott et al., this volume; Fig. 5).

Isotopic stage 2 is represented by relatively thick deposits. All cores show $\delta^{18} \mathrm{O}$ values above $+4 \%$ between approximately 2.5 and 4 mbsf. These values reflect complete glacial conditions and near-standard, marine surface water (i.e., salinity near $35 \%$ ). These conditions apparently became dominant slightly before 30,000 yr b.p., as indicated by an AMS ${ }^{14} \mathrm{C}$ age of $30,280 \pm 230$ yr b.p. at about $2.5 \mathrm{~m}$ in Core 76-029-033 (Fig. 6). The isotopic stage 3 /stage 2 transition seems well marked in all profiles and 
Table 2. Nitrogen and carbon concentrations and isotopic compositions of organic matter in samples from Holes 645A, 645B, 645C, 645F, and 645G.

\begin{tabular}{|c|c|c|c|c|c|}
\hline Core/section & $\begin{array}{l}\text { Depth } \\
\text { (cm) }\end{array}$ & $\delta^{15} \mathrm{~N}$ & $\begin{array}{c}\delta^{13} \mathrm{C} \\
\text { (PDB) }\end{array}$ & $\underset{(\%)}{N}$ & $\underset{(\%)}{C}$ \\
\hline $5-645 \mathrm{~A}-1-1$ & $02-03$ & 9.44 & -21.09 & 0.06 & 0.49 \\
\hline $645 \mathrm{~B}-1-1$ & $18-19$ & 6.40 & -24.64 & 0.01 & 0.13 \\
\hline $645 \mathrm{~B}-1-1$ & $39-40$ & 8.87 & -24.52 & 0.02 & 0.24 \\
\hline $645 \mathrm{~B}-1-1$ & $50-53$ & 7.27 & -25.49 & 0.03 & 0.12 \\
\hline $645 \mathrm{~B}-1-1$ & $62-63$ & 4.23 & -26.35 & 0.01 & 0.20 \\
\hline $645 \mathrm{~B}-1-1$ & $80-81$ & 6.83 & -23.46 & 0.02 & 0.25 \\
\hline $645 \mathrm{~B}-1-1$ & $100-101$ & 6.79 & -25.99 & 0.16 & 0.22 \\
\hline $645 \mathrm{~B}-1-1$ & $117-118$ & 4.97 & -23.66 & 0.03 & 0.38 \\
\hline $645 \mathrm{~B}-1-1$ & $139-140$ & 7.42 & -23.18 & 0.03 & 0.32 \\
\hline $645 \mathrm{~B}-1-2$ & 03-04 & 4.98 & -23 & 0.02 & 0.16 \\
\hline $645 \mathrm{~B}-1-2$ & $20-21$ & 7.15 & -23.80 & 0.02 & 0.20 \\
\hline $645 \mathrm{~B}-1-2$ & $39-40$ & 3.61 & & 0.02 & 0.15 \\
\hline $645 \mathrm{~B}-1-2$ & $50-53$ & 5.19 & -22.69 & 0.02 & 0.15 \\
\hline $645 \mathrm{~B}-1-2$ & $62-63$ & 4.26 & -23.20 & 0.02 & 0.25 \\
\hline $645 \mathrm{~B}-1-2$ & $80-81$ & 6.97 & -26.81 & 0.01 & 0.08 \\
\hline 645B-1-2 & $99-100$ & 5.93 & -25.17 & 0.02 & 0.20 \\
\hline $645 \mathrm{~B}-1-2$ & $119-12$ & 7.63 & -26 & 0.03 & 0.20 \\
\hline $645 \mathrm{~B}-1-2$ & $136-137$ & 8.23 & -23.73 & 0.04 & 0.12 \\
\hline $645 \mathrm{~B}-1-3$ & $50-53$ & 5.08 & & 0.01 & 0.12 \\
\hline $645 \mathrm{G}-1-1$ & $09-10$ & 9.20 & -26.52 & 0.04 & 0.16 \\
\hline $645 \mathrm{G}-1$. & $28-2$ & 7.44 & & 0.02 & .19 \\
\hline $645 \mathrm{G}-1-1$ & $49-50$ & 8.84 & -24.94 & 0.01 & 0.11 \\
\hline $645 \mathrm{G}-1-1$ & $69-70$ & 5.80 & -25.54 & 0.01 & 0.11 \\
\hline $645 \mathrm{G}-1-1$ & 93-94 & 5.28 & -25 & 0.01 & 0.13 \\
\hline $645 \mathrm{G}-1-2$ & 03-04 & 6.1 & 59 & 0.02 & .33 \\
\hline $645 \mathrm{G}-1-2$ & $20-21$ & 5.76 & -26 & 0.01 & 0.23 \\
\hline $645 \mathrm{G}-1-2$ & $40-41$ & 5.13 & 79 & 0.01 & .23 \\
\hline $645 \mathrm{G}-1-2$ & $59-60$ & 4.76 & -25 & 0.01 & 0.20 \\
\hline $645 \mathrm{G}-1-2$ & $80-8$ & 4.45 & -24 & 0.01 & .24 \\
\hline $645 \mathrm{G}-1-2$ & $99-1$ & 5.54 & -23.35 & 0.02 & 0.22 \\
\hline $645 \mathrm{G}-1-2$ & $119-1$ & 7.29 & -24 & 0.02 & .30 \\
\hline $645 \mathrm{G}-1-2$ & $8-13$ & 3.34 & -22 & 0.01 & .06 \\
\hline $645 \mathrm{G}-1-3$ & 0 & 8.6 & -24 & 0.03 & 0.31 \\
\hline $645 \mathrm{G}-1-3$ & $0-21$ & 7.36 & -22 & 0.03 & 0.26 \\
\hline $645 \mathrm{G}-1-3$ & $9-40$ & 5.64 & -26 & 0.03 & .22 \\
\hline $645 \mathrm{G}-1-3$ & $59-60$ & 7.10 & -26 & 0.02 & 0.19 \\
\hline $645 \mathrm{G}-1-3$ & $79-8$ & 6.61 & -27 & 0.01 & 0.28 \\
\hline $645 \mathrm{G}-1-3$ & $99-1 C$ & 7.58 & -23 & 0.02 & .25 \\
\hline $645 \mathrm{G}-1-3$ & $120-12$ & 10.03 & -25 & 0.04 & 0.21 \\
\hline $645 \mathrm{G}-1-4$ & $10-11$ & 5.71 & -27.12 & 0.02 & 0.45 \\
\hline $645 \mathrm{G}-1-4$ & $28-29$ & 8.4 & -25 & 0.03 & 0.54 \\
\hline $645 \mathrm{G}-1-4$ & $48-4$ & 6.31 & -27.18 & 0.03 & 0.38 \\
\hline $645 \mathrm{G}-1-4$ & 6 & 5.35 & -26 & 0.02 & 0.18 \\
\hline $645 \mathrm{G}-1-4$ & $89-90$ & 6.24 & -27 & 0.01 & .21 \\
\hline $645 \mathrm{G}-1-4$ & $109-110$ & 5.39 & -27 & 0.01 & 0.17 \\
\hline $645 \mathrm{G}-1-4$ & $129-1$ & 3.05 & -25.46 & 0.02 & 0.11 \\
\hline $645 \mathrm{G}-1-4$ & $146-147$ & 5.69 & -27 & 0.02 & 0.37 \\
\hline $645 \mathrm{G}-1-5$ & & 3.60 & -26 & 0.01 & .22 \\
\hline $645 \mathrm{G}-1-5$ & $20-21$ & 7.38 & -25 . & 0.02 & 0.19 \\
\hline $645 \mathrm{G}-1-5$ & $41-42$ & 7.01 & -23.03 & 0.03 & 0.25 \\
\hline & & & & & \\
\hline & & & 2 & 0.03 & 0.33 \\
\hline & & & & & 0.09 \\
\hline 64 & 6 & & -2 & $0 . C$ & 0.35 \\
\hline & & & & & 0.31 \\
\hline 64 & 9 & & -23 & 0. & 0.77 \\
\hline & & & & & 0.29 \\
\hline & & & -2 & & 0.12 \\
\hline & & & & & \\
\hline & & & -25 & & \\
\hline & & & & & 0.19 \\
\hline & & & & & .13 \\
\hline & & & & & \\
\hline & & & & & 17 \\
\hline & & & & & 0.40 \\
\hline & $118-1$ & & & & 0.11 \\
\hline & & & & & \\
\hline $645 \mathrm{~B}-2-4$ & $41-42$ & 10.54 & & 0.02 & 0.46 \\
\hline $645 \mathrm{~B}-2-4$ & $51-53$ & 3.81 & 23.23 & 0.02 & \\
\hline & & & & & \\
\hline & & & & 0.02 & 0.36 \\
\hline & & & -25.19 & 0.02 & 0.24 \\
\hline $645 \mathrm{~F}-2-1$ & $70-71$ & 5.77 & -22.88 & 0.02 & 0.20 \\
\hline
\end{tabular}

Table 2 (continued).

\begin{tabular}{|c|c|c|c|c|c|}
\hline Core/section & $\begin{array}{l}\text { Depth } \\
\text { (cm) }\end{array}$ & $\delta^{15} \mathrm{~N}$ & $\begin{array}{c}\delta^{13} \mathrm{C} \\
\text { (PDB) }\end{array}$ & $\begin{array}{c}N \\
(\%)\end{array}$ & $\begin{array}{c}C \\
(\%)\end{array}$ \\
\hline $645 \mathrm{~F}-2-1$ & $87-88$ & 6.47 & -24.91 & 0.01 & 0.35 \\
\hline $645 \mathrm{G}-2-1$ & $58-59$ & 4.42 & -27.09 & 0.02 & 0.33 \\
\hline $645 \mathrm{G}-2-1$ & $79-80$ & 4.47 & -24.68 & 0.02 & 0.35 \\
\hline $645 \mathrm{G}-2-1$ & $100-101$ & 7.36 & -25.67 & 0.03 & 0.26 \\
\hline $645 G-2-1$ & $120-121$ & 4.84 & -25.84 & 0.02 & 0.05 \\
\hline $645 \mathrm{G}-2-1$ & $140-141$ & 7.97 & -26.41 & 0.03 & 0.27 \\
\hline $645 \mathrm{~F}-3-1$ & 04-05 & 4.82 & -23.09 & 0.02 & 0.29 \\
\hline $645 \mathrm{~F}-3-1$ & $21-22$ & 5.05 & -25.62 & 0.02 & 0.30 \\
\hline 645F-3-1 & $39-40$ & 4.82 & -23.04 & 0.03 & 0.41 \\
\hline $645 \mathrm{~F}-3-1$ & $59-60$ & 6.33 & -24.20 & 0.02 & 0.24 \\
\hline $645 \mathrm{~F}-3-1$ & $80-81$ & 8.28 & -25.74 & 0.03 & 0.17 \\
\hline $645 \mathrm{~F}-3-1$ & $100-101$ & 4.24 & -24.20 & 0.02 & 0.38 \\
\hline $645 \mathrm{~F}-3-1$ & $119-120$ & 3.61 & -21.03 & 0.03 & 0.05 \\
\hline $645 \mathrm{~F}-3-1$ & $142-143$ & 6.37 & -24.97 & 0.02 & 0.32 \\
\hline $645 \mathrm{~F}-3-2$ & $10-11$ & & -23.66 & 0.02 & 0.12 \\
\hline $645 \mathrm{~F}-3-2$ & $30-31$ & 4.29 & -24.70 & 0.02 & 0.27 \\
\hline $645 F-3-2$ & $50-51$ & 7.17 & -26.75 & 0.02 & 0.59 \\
\hline $645 \mathrm{~F}-3-2$ & $70-71$ & 4.53 & -23.79 & 0.02 & 0.93 \\
\hline $645 F-3-2$ & $91-93$ & 5.79 & -26.28 & 0.02 & 1.01 \\
\hline $645 \mathrm{~F}-3-2$ & $110-111$ & 10.69 & -24.68 & 0.03 & 0.24 \\
\hline $645 F-3-2$ & $129-130$ & 4.76 & -22.65 & 0.04 & 0.61 \\
\hline $645 \mathrm{~F}-3-2$ & $146-147$ & 3.87 & -26.71 & 0.02 & 0.39 \\
\hline $645 F-3-3$ & $09-1$ & 3.47 & -25.16 & 0.03 & 0.89 \\
\hline $645 \mathrm{~F}-3$ & $33-$ & 4.31 & -24.15 & 0.03 & 0.38 \\
\hline $645 \mathrm{~F}-3-3$ & $50-51$ & 5.63 & -25.99 & 0.02 & 0.24 \\
\hline $645 \mathrm{~F}-3-3$ & 69-70 & 8.23 & -26.64 & 0.01 & 0.51 \\
\hline $645 \mathrm{~F}-3-3$ & $89-90$ & 4.07 & -25.51 & 0.03 & 0.72 \\
\hline $645 \mathrm{~F}-3-3$ & $109-110$ & 4.77 & -26.12 & 0.06 & 0.35 \\
\hline $645 \mathrm{~F}-3-3$ & $130-131$ & 5.37 & -21.52 & 0.02 & 0.68 \\
\hline $645 \mathrm{~F}-3-4$ & $30-31$ & & -26.74 & 0.03 & 0.65 \\
\hline $645 \mathrm{~F}-3-4$ & $52-53$ & 4.54 & -24.35 & 0.03 & 0.56 \\
\hline $5 F-3-4$ & $69-70$ & 5.00 & -25.15 & 0.03 & 0.45 \\
\hline $645 \mathrm{~F}-3-4$ & $89-90$ & 6.22 & -25.52 & 0.03 & 0.42 \\
\hline $645 \mathrm{~F}-3-4$ & $114-115$ & 5.01 & -25.84 & 0.04 & 0.42 \\
\hline $645 F-3-5$ & $09-10$ & 4.76 & -25.52 & 0.04 & 0.97 \\
\hline $645 F-3-5$ & $30-$ & 7.65 & -26.39 & 0.03 & 0.49 \\
\hline $645 F-3-5$ & $51-51$ & 4.91 & -26.33 & 0.05 & 1.20 \\
\hline $5 \mathrm{~F}_{-3-5}$ & 69-70 & 4.51 & -23.07 & 0.03 & 0.31 \\
\hline FF-3-5 & $86-87$ & 4.47 & -25.55 & 0.03 & 0.40 \\
\hline $645 F-3-5$ & $109-110$ & & -26.23 & 0.02 & 0.25 \\
\hline $645 F-3-5$ & $129-130$ & 7.22 & -26.18 & 0.03 & 0.32 \\
\hline & & & & & \\
\hline $645 C-3-3$ & & & & 0.02 & 0.28 \\
\hline $645 C-3-4$ & & & -25.46 & 0.03 & 0.42 \\
\hline & & & -25.62 & 0.02 & 0.28 \\
\hline $645 C-3-4$ & & & -24.72 & 0.02 & 0.33 \\
\hline$C-3-5$ & & & -24.22 & 0.02 & 0.35 \\
\hline $645 C-$ & & 5.87 & -27.23 & 0.01 & 0.24 \\
\hline & & 6.31 & -26.44 & 0.01 & 0.20 \\
\hline $645 C-3-5$ & 94-95 & 4.52 & -24.42 & 0.03 & 0.54 \\
\hline
\end{tabular}

has a sharp downcore shift to lower values $\left(\delta^{18} \mathrm{O}<3.0 \%\right)$ at about 4.5-5.0 mbsf in Cores 105-645G-1H (Fig. 4) and 85-027016 (Fig. 5), and slightly above 4 mbsf in Core 76-029-033 (Fig. 6). Some uncertainties still exist about the age of the global isotopic stage 3/stage 2 transition (see Fillon and Duplessy, 1980). Shackleton and Opdyke (1973) interpolated an age of about 32,000 yr b.p., which agrees with the Baffin Bay record (Fig. 8), and stated that the transition apparently occurred shortly before 30,000 yr b.p. Because the isotopic record of Baffin Bay depends strongly on the local ice budget, however, the age of the stage 3/stage 2 transition may be slightly different in Baffin Bay than at the global scale. Nevertheless, the few AMS ${ }^{14} \mathrm{C}$ dates obtained allowed us to interpolate high sedimentation rates (about $15-20 \mathrm{~cm} / \mathrm{k} . \mathrm{y}$.) during stage 2 .

Isotopic stage 3 appears well bounded by sharp shifts of $\delta^{18} \mathrm{O}$ in Cores 105-645G-1H and 85-027-016. This stage shows a succession of light peaks in the ${ }^{18} \mathrm{O}$ record (Table 1). The earliest peak, in the lower part of stage 3 , is particularly well recorded, even in the "screened" profiles of Figure 7 , with $\delta^{18} \mathrm{O}$ values as 
Table 3. Palynomorph concentrations for Holes 645A, 645B, 645C, 645F, 645G, and Cores 85-027-016TWC and 85-027016P.

\begin{tabular}{|c|c|c|c|c|c|c|c|c|c|c|}
\hline \multirow{2}{*}{$\begin{array}{l}\text { Laboratory } \\
\text { number }\end{array}$} & \multirow[b]{2}{*}{ Core/section } & \multirow{2}{*}{$\begin{array}{l}\text { Depth } \\
(\mathrm{cm})\end{array}$} & \multirow[b]{2}{*}{ Dinocysts } & \multirow[b]{2}{*}{ Pollen } & \multirow[b]{2}{*}{ Pinus } & \multirow[b]{2}{*}{ Spores } & & Reworked & palynomorphs & \\
\hline & & & & & & & Spores & Bisaccates & Angiosperms & Dinocysts \\
\hline UQP-32-1 & $105-645 \mathrm{~A}-1 \mathrm{H}-1$ & $0-02$ & 475 & 31 & 16 & - & 14 & - & 10 & - \\
\hline UQP-17-5 & $645 \mathrm{~B}-1 \mathrm{X}-1$ & $16-18$ & 49 & 28 & - & - & - & - & - & - \\
\hline UQP-17-6 & $645 \mathrm{~B}-1 \mathrm{X}-1$ & $37-39$ & - & 5 & 5 & 5 & 5 & 5 & - & - \\
\hline UQP-17-7 & $645 \mathrm{~B}-1 \mathrm{X}-1$ & $57-61$ & 11 & 11 & 11 & - & 6 & - & - & 6 \\
\hline UQP-17-8 & $645 B-1 X-1$ & $78-80$ & - & 16 & 12 & - & 95 & 24 & 6 & 6 \\
\hline UQP-18-1 & $645 \mathrm{~B}-1 \mathrm{X}-1$ & 97-99 & 7 & 7 & - & - & 23 & 31 & 8 & - \\
\hline UQP-18-3 & $645 \mathrm{~B}-1 \mathrm{X}-1$ & $115-117$ & - & 70 & 40 & 10 & 418 & 289 & 99 & 90 \\
\hline UQP-18-2 & $645 \mathrm{~B}-1 \mathrm{X}-1$ & $137-139$ & 6 & 6 & 6 & - & 19 & 7 & - & - \\
\hline UQP-20-3 & $645 \mathrm{~B}-1 \mathrm{X}-2$ & $01-03$ & - & 8 & 4 & - & 8 & 25 & 8 & 8 \\
\hline UQP-20-4 & $645 \mathrm{~B}-1 \mathrm{X}-2$ & $18-20$ & - & 7 & 4 & - & 23 & 31 & 8 & 8 \\
\hline UQP-20-5 & $645 \mathrm{~B}-1 \mathrm{X}-2$ & $37-39$ & 8 & 75 & 58 & - & 25 & 67 & 25 & 25 \\
\hline UQP-20-6 & $645 \mathrm{~B}-1 \mathrm{X}-2$ & $58-60$ & 9 & 27 & 27 & - & 18 & 54 & - & 9 \\
\hline UQP-20-7 & $645 \mathrm{~B}-1 \mathrm{X}-2$ & $78-80$ & - & 10 & 4 & - & - & - & - & 10 \\
\hline UQP-20-8 & $645 \mathrm{~B}-1 \mathrm{X}-2$ & $98-100$ & - & 9 & 6 & - & - & 36 & 9 & 18 \\
\hline UQP-21-1 & $645 B-1 X-2$ & $117-119$ & 14 & 178 & 72 & - & 100 & 100 & 114 & 64 \\
\hline UQP-21-2 & $645 \mathrm{~B}-1 \mathrm{X}-2$ & $136-138$ & 9 & 141 & 84 & 18 & 272 & 291 & 272 & - \\
\hline UQP-27-5 & $645 \mathrm{G}-1 \mathrm{H}-1$ & 07-09 & - & 31 & 11 & - & 57 & 11 & 11 & 21 \\
\hline UQP-27-6 & $645 \mathrm{G}-1 \mathrm{H}-1$ & $26-28$ & - & 29 & 19 & - & 57 & 28 & 57 & 28 \\
\hline UQP-27-7 & $645 \mathrm{G}-1 \mathrm{H}-1$ & $47-49$ & - & 10 & 10 & 10 & 20 & 10 & 21 & - \\
\hline UQP-27-8 & $645 \mathrm{G}-1 \mathrm{H}-1$ & $67-69$ & - & 9 & 5 & - & 14 & - & - & 5 \\
\hline UQP-28-1 & $645 \mathrm{G}-1 \mathrm{H}-1$ & $91-93$ & - & 16 & 16 & - & 121 & 85 & 12 & 170 \\
\hline UQP-28-2 & $645 \mathrm{G}-1 \mathrm{H}-2$ & $01-03$ & - & 22 & 22 & - & 66 & 110 & 22 & 44 \\
\hline UQP-28-3 & $645 \mathrm{G}-1 \mathrm{H}-2$ & $18-20$ & - & 53 & 26 & - & 40 & 107 & - & 13 \\
\hline UQP-28-4 & $645 \mathrm{G}-1 \mathrm{H}-2$ & $38-40$ & - & 64 & 42 & - & 53 & 53 & 11 & - \\
\hline UQP-28-5 & $645 \mathrm{G}-1 \mathrm{H}-2$ & $57-59$ & - & 31 & 31 & 10 & 89 & 50 & 10 & 30 \\
\hline UQP-26-6 & $645 \mathrm{G}-1 \mathrm{H}-2$ & $78-80$ & 9 & 39 & 39 & 39 & 59 & 208 & 50 & 10 \\
\hline UQP-28-7 & $645 \mathrm{G}-1 \mathrm{H}-2$ & $97-99$ & 59 & 302 & 227 & 84 & 209 & 167 & 109 & 16 \\
\hline UQP-28-8 & $645 \mathrm{G}-1 \mathrm{H}-2$ & $117-119$ & 29 & 171 & 114 & 38 & 67 & 157 & 67 & 48 \\
\hline UQP-29-1 & $645 \mathrm{G}-1 \mathrm{H}-2$ & $136-138$ & 9 & 171 & 81 & 36 & 36 & 45 & 63 & - \\
\hline UQP-29-2 & $645 \mathrm{G}-1 \mathrm{H}-3$ & $01-03$ & 9 & 45 & 36 & 9 & 72 & 63 & 18 & 18 \\
\hline UQP-29-3 & $645 \mathrm{G}-1 \mathrm{H}-3$ & $18-20$ & - & 60 & 30 & - & 90 & 60 & - & 15 \\
\hline UQP-29-4 & $645 \mathrm{G}-1 \mathrm{H}-3$ & $37-39$ & 7 & 15 & 15 & - & 28 & 28 & 7 & 7 \\
\hline UQP-29-5 & $645 \mathrm{G}-1 \mathrm{H}-3$ & $57-59$ & 39 & 66 & 57 & 39 & 58 & 9 & 9 & - \\
\hline UQP-29-6 & $645 \mathrm{G}-1 \mathrm{H}-3$ & $76-79$ & 9 & 78 & 45 & 9 & 55 & 37 & 18 & 9 \\
\hline UQP-29-7 & $645 \mathrm{G}-1 \mathrm{H}-3$ & $97-99$ & 9 & 19 & 9 & - & 46 & 55 & - & 46 \\
\hline UQP-29-8 & $645 \mathrm{G}-1 \mathrm{H}-3$ & $118-120$ & - & 25 & 18 & - & 27 & 9 & - & 9 \\
\hline UQP-30-1 & $645 \mathrm{G}-1 \mathrm{H}-4$ & 08-10 & 29 & 12 & 9 & 9 & 9 & 9 & 18 & - \\
\hline UQP-30-2 & $645 \mathrm{G}-1 \mathrm{H}-4$ & $26-28$ & 20 & - & - & - & - & - & 20 & - \\
\hline UQP-30-3 & $645 \mathrm{G}-1 \mathrm{H}-4$ & $46-48$ & 8 & 16 & 8 & - & 56 & 24 & 32 & - \\
\hline UQP-30-4 & $645 \mathrm{G}-1 \mathrm{H}-4$ & $67-69$ & 8 & 16 & 8 & 8 & 96 & 80 & 8 & 24 \\
\hline UQP-30-5 & $645 \mathrm{G}-1 \mathrm{H}-4$ & $87-89$ & - & 6 & 6 & - & - & 9 & - & 18 \\
\hline UQP-30-6 & $645 \mathrm{G}-1 \mathrm{H}-4$ & $107-109$ & 9 & 19 & 19 & - & - & 19 & 9 & - \\
\hline UQP-30-7 & $645 \mathrm{G}-1 \mathrm{H}-4$ & $127-129$ & 47 & 3 & 3 & - & 18 & 18 & - & - \\
\hline UQP-30-8 & $645 \mathrm{G}-1 \mathrm{H}-4$ & $144-146$ & 145 & 45 & 18 & 9 & 54 & - & - & - \\
\hline UQP-31-1 & $645 \mathrm{G}-1 \mathrm{H}-5$ & $01-03$ & 51 & 28 & 20 & - & 26 & - & - & - \\
\hline UQP-31-2 & $645 \mathrm{G}-1 \mathrm{H}-5$ & $18-20$ & 23 & 61 & 23 & 8 & 92 & 107 & 23 & 31 \\
\hline UQP-31-3 & $645 \mathrm{G}-1 \mathrm{H}-5$ & $39-41$ & - & 22 & 9 & - & 141 & 66 & - & 28 \\
\hline UQP-18-4 & $645 \mathrm{~B}-2 \mathrm{X}-2$ & $28-30$ & 75 & 225 & 128 & - & 165 & 150 & 38 & 53 \\
\hline UQP-18-5 & $645 \mathrm{~B}-2 \mathrm{X}-2$ & $58-60$ & 161 & 472 & 252 & 95 & 521 & 170 & 95 & 85 \\
\hline UQP-18-6 & $645 \mathrm{~B}-2 \mathrm{X}-2$ & $78-80$ & 48 & 62 & 28 & - & 192 & 68 & 28 & 21 \\
\hline UQP-18-7 & $645 \mathrm{~B}-2 \mathrm{X}-2$ & $94-96$ & - & 42 & 25 & - & 185 & 109 & 51 & 17 \\
\hline UQP-18-8 & $645 \mathrm{~B}-2 \mathrm{X}-2$ & $117-119$ & 9 & 36 & 18 & - & 198 & 198 & 45 & 18 \\
\hline UQP-19-1 & $645 \mathrm{~B}-2 \mathrm{X}-2$ & $138-140$ & 18 & 37 & 9 & - & 198 & 171 & 90 & 9 \\
\hline UQP-19-2 & $645 \mathrm{~B}-2 \mathrm{X}-3$ & $01-03$ & 17 & 53 & 27 & - & 239 & 106 & 150 & 8 \\
\hline UQP-19-3 & $645 \mathrm{~B}-2 \mathrm{X}-3$ & $18-20$ & 8 & 44 & 16 & - & 273 & 202 & 185 & 8 \\
\hline UQP-19-4 & $645 \mathrm{~B}-2 \mathrm{X}-3$ & $38-40$ & 57 & 19 & 9 & - & 304 & 152 & 57 & - \\
\hline UQP-19-5 & $534 \mathrm{~B}-2 \mathrm{X}-3$ & $59-60$ & 18 & 76 & 29 & - & 180 & 180 & 37 & 18 \\
\hline UQP-19-6 & $645 \mathrm{~B}-2 \mathrm{X}-3$ & $77-79$ & 19 & 57 & 9 & - & 228 & 181 & 114 & 19 \\
\hline UQP-19-7 & $645 \mathrm{~B}-2 \mathrm{X}-3$ & $97-99$ & - & 46 & 9 & - & 184 & 111 & 120 & 9 \\
\hline UQP-19-8 & $645 \mathrm{~B}-2 \mathrm{X}-3$ & $115-117$ & 28 & 47 & 18 & 9 & 149 & 177 & 102 & 28 \\
\hline UQP-20-1 & $645 \mathrm{~B}-2 \mathrm{X}-4$ & $18-20$ & 8 & 26 & 8 & - & 96 & 139 & 87 & 8 \\
\hline UQP-20-2 & $645 \mathrm{~B}-2 \mathrm{X}-4$ & $38-40$ & - & 76 & 47 & - & 114 & 123 & 105 & 9 \\
\hline UQP-22-4 & $645 \mathrm{~F}-2 \mathrm{H}-1$ & $07-09$ & 16 & 104 & 56 & - & 144 & 161 & 104 & 56 \\
\hline UQP-22-5 & $645 \mathrm{~F}-2 \mathrm{H}-1$ & $28-30$ & 14 & 68 & 49 & - & 122 & 141 & 115 & 34 \\
\hline UQP-22-6 & $645 \mathrm{~F}-2 \mathrm{H}-1$ & $50-52$ & 95 & 181 & 124 & 19 & 171 & 181 & 238 & 48 \\
\hline UQP-22-7 & $645 \mathrm{~F}-2 \mathrm{H}-1$ & $68-70$ & - & 36 & 18 & - & 234 & 334 & 36 & 72 \\
\hline UQP-22-8 & $645 \mathrm{~F}-2 \mathrm{H}-1$ & $88-90$ & 7 & 36 & 29 & - & 167 & 159 & 116 & 28 \\
\hline UQP-31-4 & $645 \mathrm{G}-2 \mathrm{H}-1$ & $56-58$ & 8 & 17 & 8 & - & 114 & 8 & 8 & 8 \\
\hline UQP-31-5 & $645 \mathrm{G}-2 \mathrm{H}-1$ & $77-79$ & 112 & 271 & 178 & 159 & 187 & 178 & 94 & 75 \\
\hline UQP-31-6 & $645 \mathrm{G}-2 \mathrm{H}-1$ & $98-100$ & 26 & 43 & 18 & 52 & 95 & 69 & 61 & 18 \\
\hline UQP-31-7 & $645 \mathrm{G}-2 \mathrm{H}-1$ & $118-120$ & 10 & 49 & 10 & - & 175 & 146 & 58 & - \\
\hline UQP-31-8 & $645 \mathrm{G}-2 \mathrm{H}-1$ & $138-140$ & 20 & - & - & - & 108 & 49 & 20 & - \\
\hline
\end{tabular}


Table 3 (continued).

\begin{tabular}{|c|c|c|c|c|c|c|c|c|c|c|}
\hline \multirow{2}{*}{$\begin{array}{l}\text { Laboratory } \\
\text { number }\end{array}$} & \multirow[b]{2}{*}{ Core/section } & \multirow{2}{*}{$\begin{array}{l}\text { Depth } \\
(\mathrm{cm})\end{array}$} & \multirow[b]{2}{*}{ Dinocysts } & \multirow[b]{2}{*}{ Pollen } & \multirow[b]{2}{*}{ Pinus } & \multirow[b]{2}{*}{ Spores } & & Reworke & palynomorphs & \\
\hline & & & & & & & Spores & Bisaccates & Angiosperms & Dinocysts \\
\hline UQP-23-1 & $645 \mathrm{~F}-3 \mathrm{H}-1$ & $02-04$ & 18 & 98 & 89 & 9 & 301 & 274 & 124 & 80 \\
\hline UQP-23-2 & $645 \mathrm{~F}-3 \mathrm{H}-1$ & $19-21$ & 36 & 126 & 108 & 18 & 495 & 333 & 279 & 108 \\
\hline UQP-23-3 & $645 \mathrm{~F}-3 \mathrm{H}-1$ & $37-39$ & 35 & 105 & 105 & 35 & 1051 & 736 & 526 & 105 \\
\hline UQP-23-4 & $645 \mathrm{~F}-3 \mathrm{H}-1$ & $57-59$ & 31 & 94 & 63 & - & 1283 & 907 & 438 & 250 \\
\hline UQP-23-5 & $645 \mathrm{~F}-3 \mathrm{H}-1$ & $77-79$ & - & 103 & 52 & 35 & 857 & 531 & 292 & 172 \\
\hline UQP-23-6 & $645 \mathrm{~F}-3 \mathrm{H}-1$ & $98-100$ & - & 56 & 19 & 19 & 482 & 687 & 260 & 75 \\
\hline UQP-23-7 & $645 \mathrm{~F}-3 \mathrm{H}-1$ & $117-119$ & - & - & - & - & 203 & 277 & 314 & 19 \\
\hline UQP-23-8 & $645 \mathrm{~F}-3 \mathrm{H}-1$ & $140-142$ & - & 28 & 10 & - & 113 & 75 & 103 & 19 \\
\hline UQP-24-1 & $645 \mathrm{~F}-3 \mathrm{H}-2$ & 08-10 & 76 & 118 & 85 & 9 & 186 & 254 & 102 & 34 \\
\hline UQP-24-2 & $645 \mathrm{~F}-3 \mathrm{H}-2$ & $28-30$ & 8 & 39 & 16 & 16 & 94 & 86 & 71 & 16 \\
\hline UQP-24-3 & $645 \mathrm{~F}-3 \mathrm{H}-2$ & $48-50$ & 30 & 15 & 15 & - & 242 & 573 & 242 & 15 \\
\hline UQP-24-4 & $645 \mathrm{~F}-3 \mathrm{H}-2$ & $68-70$ & - & 9 & 9 & - & 545 & 1121 & 333 & - \\
\hline UQP-24-5 & $645 \mathrm{~F}-3 \mathrm{H}-2$ & $88-90$ & - & 7 & - & 8 & 324 & 697 & 274 & 100 \\
\hline UQP-24-6 & $645 \mathrm{~F}-3 \mathrm{H}-2$ & $108-110$ & 58 & 39 & 29 & - & 232 & 174 & 78 & 39 \\
\hline UQP-24-7 & $645 \mathrm{~F}-3 \mathrm{H}-2$ & $127-129$ & - & 10 & 10 & - & 865 & 1262 & 541 & 180 \\
\hline UQP-24-8 & $645 \mathrm{~F}-3 \mathrm{H}-2$ & $144-146$ & - & - & - & - & 151 & 322 & 57 & - \\
\hline UQP-25-1 & $645 \mathrm{~F}-3 \mathrm{H}-3$ & $07-09$ & - & 8 & 8 & - & 331 & 304 & 110 & 28 \\
\hline UQP-25-2 & $645 \mathrm{~F}-3 \mathrm{H}-3$ & $31-33$ & 120 & 3 & 3 & - & 130 & 60 & 30 & 10 \\
\hline UQP-25-3 & $645 \mathrm{~F}-3 \mathrm{H}-3$ & $48-50$ & - & 6 & 6 & - & 369 & 111 & 480 & - \\
\hline UQP-25-4 & $645 \mathrm{~F}-3 \mathrm{H}-3$ & $68-70$ & 13 & - & - & - & 153 & 115 & 64 & 13 \\
\hline UQP-25-5 & $645 \mathrm{~F}-3 \mathrm{H}-3$ & $87-89$ & 20 & - & - & - & 259 & 378 & 100 & 80 \\
\hline UQP-25-6 & $645 \mathrm{~F}-3 \mathrm{H}-3$ & $107-109$ & 15 & - & - & - & 126 & 415 & 108 & 36 \\
\hline UQP-25-7 & $645 \mathrm{~F}-3 \mathrm{H}-3$ & $128-130$ & - & - & - & - & 113 & 99 & 42 & - \\
\hline UQP-25-8 & $645 \mathrm{~F}-3 \mathrm{H}-4$ & 07-09 & 11 & 33 & 33 & - & 165 & 264 & 176 & 11 \\
\hline UQP-26-1 & $645 \mathrm{~F}-3 \mathrm{H}-4$ & $28-30$ & 17 & 85 & 51 & - & 273 & 597 & 188 & 34 \\
\hline UQP-26-2 & $645 \mathrm{~F}-3 \mathrm{H}-4$ & $50-52$ & 146 & 9 & 9 & - & 480 & 335 & 262 & 73 \\
\hline UQP-26-3 & $645 \mathrm{~F}-3 \mathrm{H}-4$ & $67-69$ & - & - & - & - & 518 & 291 & 388 & - \\
\hline UQP-26-4 & $645 \mathrm{~F}-3 \mathrm{H}-4$ & $87-89$ & - & 93 & 93 & - & 558 & 93 & 217 & 31 \\
\hline UQP-26-5 & $645 \mathrm{~F}-3 \mathrm{H}-4$ & $112-114$ & 81 & 28 & 28 & 14 & 387 & 111 & 194 & 27 \\
\hline UQP-26-6 & $645 \mathrm{~F}-3 \mathrm{H}-5$ & $08-10$ & 127 & 39 & 10 & - & 627 & 98 & 108 & 88 \\
\hline UQP-26-7 & $645 \mathrm{~F}-3 \mathrm{H}-5$ & $28-30$ & - & 16 & 8 & - & 138 & 293 & 57 & - \\
\hline UQP-26-8 & $645 \mathrm{~F}-3 \mathrm{H}-5$ & $48-50$ & - & 9 & 9 & - & 191 & 272 & 163 & - \\
\hline UQP-27-1 & $645 \mathrm{~F}-3 \mathrm{H}-5$ & $67-69$ & - & - & - & - & 625 & 184 & 220 & - \\
\hline UQP-27-2 & $645 \mathrm{~F}-3 \mathrm{H}-5$ & $84-86$ & - & - & - & 19 & 303 & - & - & 38 \\
\hline UQP-27-3 & $645 \mathrm{~F}-3 \mathrm{H}-5$ & $107-109$ & 16 & - & - & - & 213 & 82 & 16 & 49 \\
\hline UQP-27-4 & $645 \mathrm{~F}-3 \mathrm{H}-5$ & $128-130$ & - & - & - & - & 306 & 111 & 84 & - \\
\hline UQP-21-3 & $645 \mathrm{C}-3 \mathrm{H}-3$ & $108-110$ & 60 & 90 & 90 & 30 & 931 & 391 & 210 & 30 \\
\hline UQP-21-4 & $645 \mathrm{C}-3 \mathrm{H}-3$ & $130-132$ & 56 & 18 & 6 & - & 798 & 204 & 93 & 93 \\
\hline UQP-21-5 & $645 \mathrm{C}-3 \mathrm{H}-4$ & $01-03$ & 18 & 36 & 9 & - & 298 & 54 & 9 & 90 \\
\hline UQP-21-6 & $645 \mathrm{C}-3 \mathrm{H}-4$ & $19-21$ & 702 & 31 & - & 10 & 126 & 73 & 52 & 63 \\
\hline UQP-21-7 & $645 \mathrm{C}-3 \mathrm{H}-4$ & $36-39$ & - & 18 & 9 & - & 138 & 37 & 9 & 37 \\
\hline UQP-21-8 & $645 \mathrm{C}-3 \mathrm{H}-5$ & $08-10$ & - & 9 & - & - & 18 & - & - & - \\
\hline UQP-22-1 & $645 \mathrm{C}-3 \mathrm{H}-5$ & $30-32$ & 18 & 9 & 3 & - & 27 & 9 & 9 & - \\
\hline UQP-22-2 & $645 \mathrm{C}-3 \mathrm{H}-5$ & $48-50$ & 9 & 27 & - & - & - & 38 & - & 28 \\
\hline UQP-22-3 & $645 \mathrm{C}-3 \mathrm{H}-5$ & $92-94$ & 481 & 427 & 317 & 103 & 379 & 296 & 62 & 82 \\
\hline UQP-34-8 & $645 \mathrm{C}-3 \mathrm{H}, \mathrm{CC}$ & & 304 & 389 & 328 & 128 & 249 & 49 & 36 & 78 \\
\hline UQP-76-5 & 85-027-016TWC & 5 & 1006 & 8 & 1 & - & - & 7 & 3 & - \\
\hline UQP-74-2 & & 14 & 18 & 4 & - & - & 9 & - & - & 4 \\
\hline UQP-74-3 & & 20 & 13 & 20 & 14 & - & 14 & 84 & 35 & 14 \\
\hline UQP-75-5 & & 31 & 17 & 9 & 5 & - & 55 & 74 & 46 & 9 \\
\hline UQP-74-6 & & 40 & - & 10 & 6 & - & - & 6 & 17 & - \\
\hline UQP-75-1 & & 61 & - & 27 & 27 & 9 & 110 & 119 & 18 & 9 \\
\hline UQP-75-2 & & 71 & - & - & - & - & 59 & - & - & - \\
\hline UQP-75-3 & & 81 & - & 6 & 6 & - & 15 & 37 & 7 & 15 \\
\hline UQP-75-4 & & 90 & 36 & 12 & 12 & 9 & 182 & 273 & 82 & 18 \\
\hline UQP-75-5 & & 101 & - & 60 & 37 & 18 & 37 & 266 & 110 & 18 \\
\hline UQP-75-6 & & 112 & - & 47 & 36 & 8 & 39 & 126 & 24 & 32 \\
\hline UQP-76-1 & & 120 & - & - & - & - & 5 & 5 & - & 19 \\
\hline UQP-76-2 & & 130 & 6 & 15 & 10 & 7 & 190 & 79 & 26 & 13 \\
\hline UQP-60-1 & $85-027-016 \mathrm{P}$ & 1 & 134 & 8 & - & - & - & - & - & - \\
\hline UQP-60-2 & & 10 & - & - & - & - & 15 & 5 & - & - \\
\hline UQP-60-4 & & 31 & - & 6 & 2 & - & 46 & 11 & 17 & - \\
\hline UQP-60-5 & & 44 & 80 & 20 & 9 & - & 43 & 47 & 28 & 5 \\
\hline UQP-60-6 & & 51 & - & - & - & - & 78 & 31 & 31 & - \\
\hline UQP-61-1 & & 60 & 24 & 24 & 12 & 6 & 148 & 71 & 47 & 12 \\
\hline UQP-61-2 & & 71 & - & 6 & 6 & - & 12 & - & 6 & - \\
\hline UQP-61-3 & & 80 & - & 6 & - & 6 & 12 & 18 & 12 & 6 \\
\hline UQP-61-4 & & 99 & 6 & 46 & 29 & 6 & 218 & 71 & 171 & 47 \\
\hline UQP-61-5 & & 110 & 6 & 4 & 4 & - & 47 & 107 & 24 & 59 \\
\hline UQP-61-6 & & 120 & 9 & 79 & 35 & - & 79 & 510 & 132 & 70 \\
\hline UQP-62-1 & & 131 & - & 100 & 48 & 14 & 94 & 246 & 238 & 65 \\
\hline
\end{tabular}


Table 3 (continued).

\begin{tabular}{|c|c|c|c|c|c|c|c|c|c|c|}
\hline \multirow{2}{*}{$\begin{array}{l}\text { Laboratory } \\
\text { number }\end{array}$} & \multirow[b]{2}{*}{ Core/section } & \multirow{2}{*}{$\begin{array}{l}\text { Depth } \\
(\mathrm{cm})\end{array}$} & \multirow[b]{2}{*}{ Dinocysts } & \multirow[b]{2}{*}{ Pollen } & \multirow[b]{2}{*}{ Pinus } & \multirow[b]{2}{*}{ Spores } & \multicolumn{4}{|c|}{ Reworked palynomorphs } \\
\hline & & & & & & & Spores & Bisaccates & Angiosperms & Dinocysts \\
\hline UQP-62-2 & & 140 & - & 50 & 36 & 6 & 28 & 68 & 40 & 28 \\
\hline UQP-62-3 & & 151 & 18 & 27 & 15 & - & 31 & 58 & 18 & 5 \\
\hline UQP-62-4 & & 160 & - & 20 & 20 & - & 138 & 184 & 77 & 31 \\
\hline UQP-62-5 & & 171 & 8 & 30 & 23 & - & 136 & 196 & 75 & 68 \\
\hline UQP-62-6 & & 180 & - & 76 & 52 & - & 194 & 466 & 324 & 91 \\
\hline UQP-63-1 & & 191 & 29 & 10 & 10 & 10 & 324 & 362 & 201 & 38 \\
\hline UQP-63-2 & & 200 & - & 24 & 24 & - & 505 & 409 & 241 & 72 \\
\hline UQP-63-3 & & 210 & 15 & 30 & 5 & 15 & 195 & 374 & 269 & - \\
\hline UQP-63-4 & & 221 & 18 & 92 & 61 & 46 & 128 & 165 & 101 & 37 \\
\hline UQP-63-5 & & 230 & 17 & 17 & 17 & - & 200 & 133 & 217 & 50 \\
\hline UQP-63-6 & & 241 & - & - & - & - & 99 & 41 & 41 & - \\
\hline UQP-64-1 & & 253 & 16 & 45 & 27 & 25 & 41 & 33 & 25 & 17 \\
\hline UQP-64-2 & & 262 & 36 & 27 & 9 & - & 72 & 27 & 81 & 18 \\
\hline UQP-64-3 & & 271 & 8 & - & - & - & 87 & 63 & 32 & 8 \\
\hline UQP-64-4 & & 277 & 9 & 36 & 12 & - & 92 & 119 & - & 27 \\
\hline UQP-64-5 & & 288 & - & 32 & 16 & 0 & 135 & 216 & 45 & 99 \\
\hline UQP-64-6 & & 299 & - & 21 & - & 21 & 84 & 63 & 21 & 42 \\
\hline UQP-65-1 & & 310 & - & - & - & - & 47 & 19 & 9 & - \\
\hline UQP-65-2 & & 319 & 21 & 21 & 16 & 14 & 111 & 93 & 56 & 42 \\
\hline UQP-65-3 & & 330 & 32 & 24 & 11 & - & 33 & 49 & - & 24 \\
\hline UQP-65-4 & & 341 & - & 18 & 5 & - & 97 & 9 & 9 & 9 \\
\hline UQP-65-5 & & 350 & 15 & 45 & 27 & 8 & 91 & 68 & 45 & 15 \\
\hline UQP-65-6 & & 360 & - & 7 & 7 & - & 107 & 67 & 13 & 13 \\
\hline UQP-66-1 & & 371 & - & 7 & 7 & - & 7 & - & - & - \\
\hline UQP-66-2 & & 395 & - & - & - & - & 9 & 9 & 18 & - \\
\hline UQP-66-3 & & 405 & 8 & 51 & 20 & 17 & 68 & 34 & 17 & - \\
\hline UQP-66-4 & & 416 & - & 20 & 3 & - & 120 & 34 & 77 & 17 \\
\hline UQP-66-5 & & 426 & 14 & 16 & 16 & - & 63 & 63 & 14 & 42 \\
\hline UQP-66-6 & & 436 & - & 8 & - & - & 68 & 43 & - & - \\
\hline UQP-67-1 & & 446 & - & - & - & 9 & 76 & 66 & 9 & 19 \\
\hline UQP- $67-2$ & & 455 & 78 & 14 & - & - & 135 & 85 & 14 & 28 \\
\hline UQP-67-3 & & 465 & - & - & - & - & 12 & 29 & 12 & 6 \\
\hline UQP-67-4 & & 474 & - & 7 & 7 & - & 50 & 47 & 28 & 7 \\
\hline UQP-67-5 & & 485 & - & 16 & 2 & - & 32 & 56 & 32 & 24 \\
\hline UQP-67-6 & & 494 & 18 & 24 & 15 & - & 27 & 18 & 9 & 18 \\
\hline UQP-68-1 & & 505 & - & - & - & - & 19 & - & 19 & 9 \\
\hline UQP-68-2 & & 514 & 16 & 24 & 12 & 8 & 76 & 30 & 91 & 23 \\
\hline UQP-68-3 & & 525 & 160 & 48 & 31 & 15 & 95 & 80 & 51 & 8 \\
\hline UQP-68-4 & & 534 & 8 & 8 & - & - & 16 & - & - & - \\
\hline UQP-68-5 & & 545 & - & 27 & 21 & 13 & 27 & 54 & 27 & 27 \\
\hline UQP-68-6 & & 554 & 16 & 10 & 5 & 16 & 5 & 5 & 5 & 10 \\
\hline UQP-69-1 & & 565 & 140 & - & - & - & 21 & - & - & 7 \\
\hline UQP-69-2 & & 574 & 155 & 229 & 173 & 109 & 155 & 220 & 55 & 46 \\
\hline UQP-69-3 & & 585 & 56 & 47 & 18 & 9 & 93 & 46 & 65 & 9 \\
\hline UQP-69-4 & & 594 & 9 & - & - & - & 102 & 241 & 9 & 28 \\
\hline UQP-69-5 & & 606 & - & - & - & - & 69 & 23 & - & 8 \\
\hline UQP-69-6 & & 614 & 6 & 6 & 6 & - & 93 & 25 & 12 & 6 \\
\hline UQP-70-1 & & 625 & - & 5 & - & - & 16 & 10 & - & - \\
\hline UQP-70-2 & & 634 & 8 & 8 & 8 & - & 33 & 16 & 8 & - \\
\hline UQP-70-3 & & 645 & 35 & 7 & - & 7 & 63 & 63 & 55 & 16 \\
\hline UQP-70-4 & & 654 & 129 & - & - & - & 84 & 145 & 8 & - \\
\hline UQP-70-5 & & 665 & - & - & - & - & 8 & - & - & 16 \\
\hline UQP-70-6 & & 674 & - & 20 & 15 & - & 92 & 92 & 15 & 31 \\
\hline UQP-71-1 & & 685 & - & 50 & 45 & - & 152 & 197 & 72 & 45 \\
\hline UQP-71-2 & & 694 & 24 & 84 & 25 & 25 & 302 & 319 & 151 & 17 \\
\hline UQP-71-3 & & 705 & 8 & 32 & 17 & - & 242 & 216 & 52 & 17 \\
\hline UQP-71-4 & & 714 & 1367 & 188 & 98 & 9 & 259 & 375 & 205 & 63 \\
\hline UQP-71-5 & & 725 & 20 & 40 & 19 & - & 144 & 354 & 201 & 29 \\
\hline UQP-71-6 & & 736 & - & 40 & 8 & 8 & 107 & 239 & 91 & 41 \\
\hline UQP-72-1 & & 745 & - & 29 & - & - & 236 & 148 & 87 & 59 \\
\hline UQP-72-2 & & 754 & 9 & 9 & 9 & - & 213 & 68 & 10 & 77 \\
\hline UQP-72-3 & & 765 & 9 & - & - & - & 18 & 62 & 23 & 23 \\
\hline UQP-72-4 & & 774 & - & - & - & - & 17 & 8 & 25 & 8 \\
\hline UQP-72-5 & & 785 & - & - & - & - & - & 9 & - & 9 \\
\hline UQP-72-6 & & 794 & - & 9 & - & - & 18 & - & - & - \\
\hline UQP-73-1 & & 805 & - & - & - & - & 9 & - & - & 9 \\
\hline UQP-73-2 & & 815 & 10 & - & - & - & 88 & 29 & 29 & 29 \\
\hline UQP-73-3 & & 824 & - & - & - & - & 37 & 9 & - & 9 \\
\hline
\end{tabular}

low as $\sim 1.5 \%$. This peak is observed in all ${ }^{18} \mathrm{O}$ high-resolution records from Baffin Bay (see Aksu, 1981: Cores 77-027-017 and 76-029-040) and reflects a significant ice-retreat episode, at least in Arctic Canada, during the Mid-Wisconsinan, which also was shown by continental stratigraphies (e.g., Andrews et al., 1984).
Isotopic stage 3 seems to have been characterized by lower sedimentation rates $(\sim 10 \mathrm{~cm} / \mathrm{k} . \mathrm{y}$.) than isotopic stage 2 . This is not the case in Labrador Sea (Fillon and Duplessy, 1980; de Vernal and Hillaire-Marcel, 1987), where no significant differences in sedimentation rate were observed between stages 2 and 3 . 

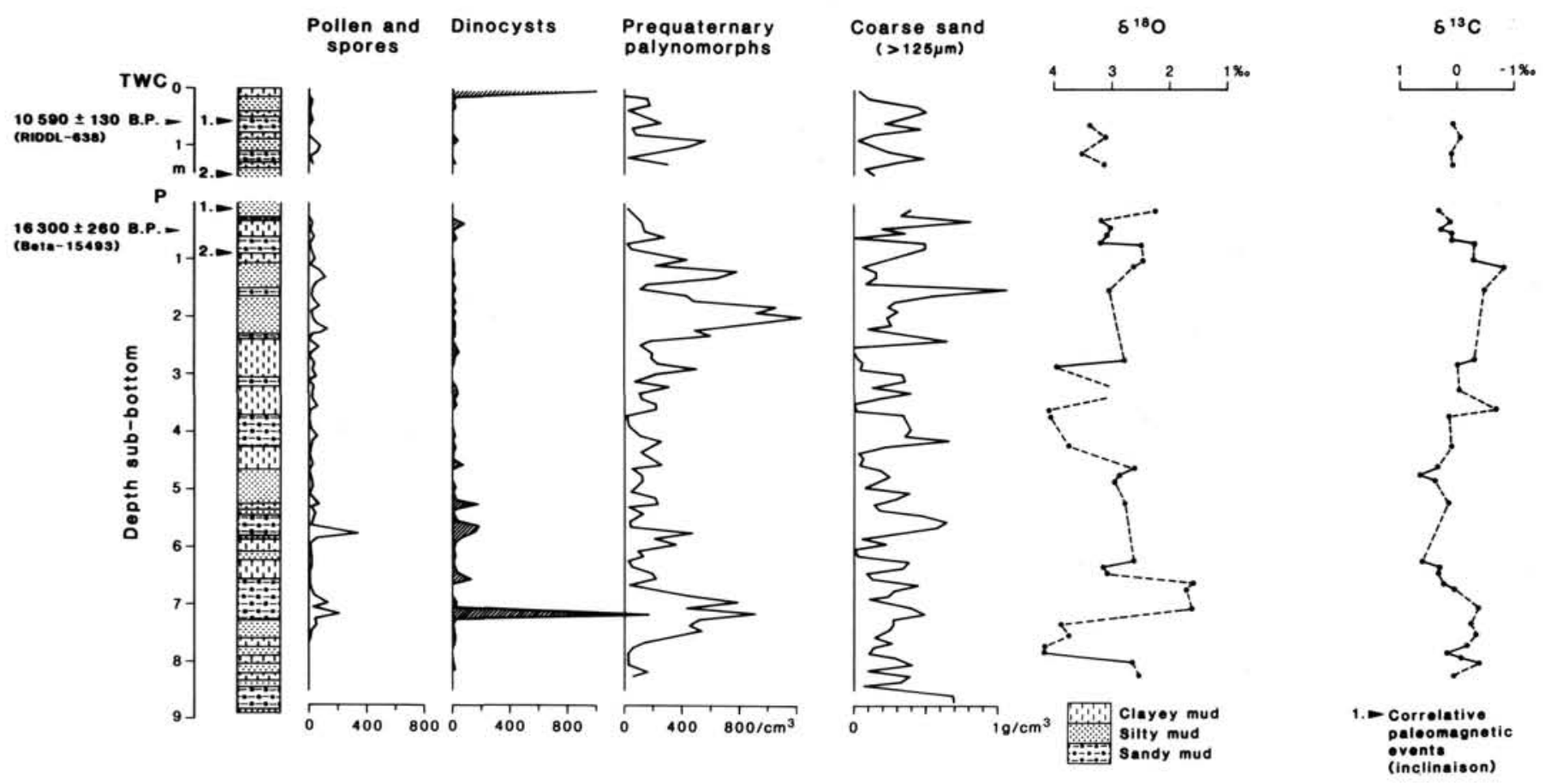

Figure 5. Schematic lithostratigraphy, palynomorph concentrations, coarse-sand concentrations, and screened $\delta^{13} \mathrm{C}$ and $\delta^{18} \mathrm{O}$ profiles in Cores $85-027$ 016TWC and 85-027-016P $\left(70^{\circ} 30^{\prime} 78 \mathrm{~N}, 64^{\circ} \mathrm{s} 31^{\prime} 24 \mathrm{~W}\right.$; water depth $\left.=2091 \mathrm{~m}\right)$. In the $\delta^{13} \mathrm{C}$ and $\delta^{18} \mathrm{O}$ curves, all data points from small populations (i.e., $<40$ individuals $/ 8 \mathrm{~cm}^{3}$ ) have been excluded. Dotted lines join data points over sample gaps. High-resolution magnetostratigraphic data (2-cm sampling intervals) from Core 85-027-016 are reported by Thouveny (in press).

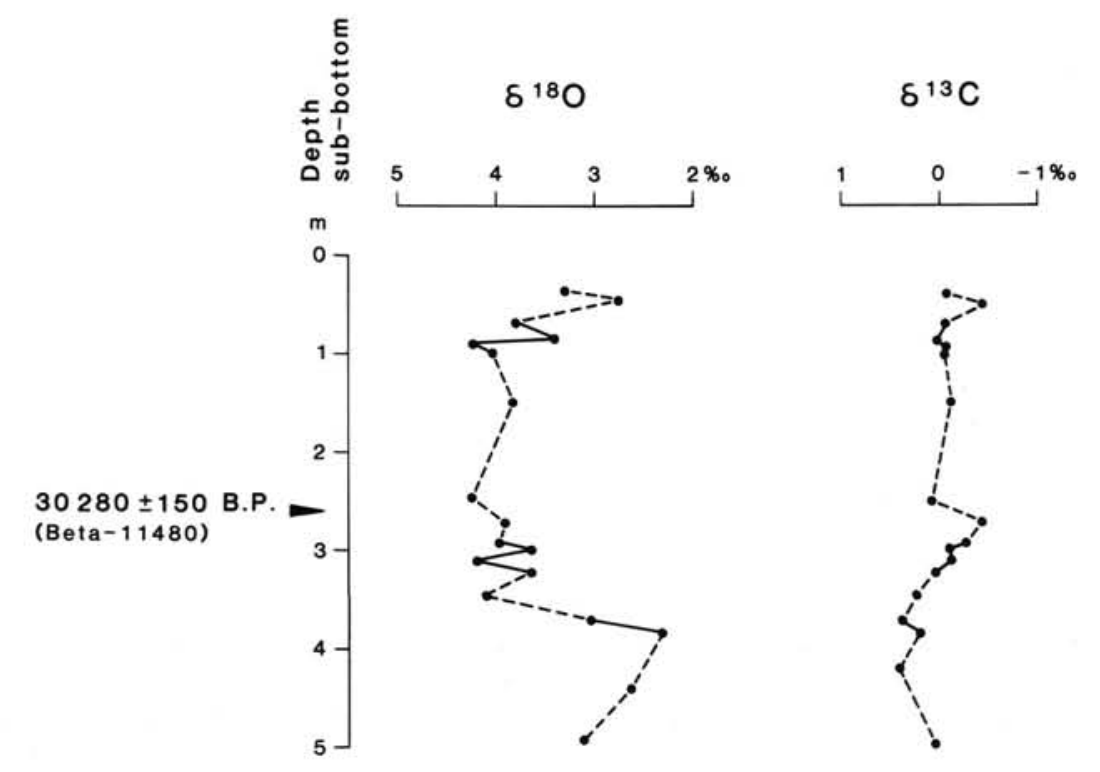

Figure 6. Screened $\delta^{13} \mathrm{C}$ and $\delta^{18} \mathrm{O}$ profiles in Core 76-029-033P $\left(71^{\circ} 20^{\prime} \mathrm{N}, 64^{\circ} 16^{\prime} \mathrm{W}\right.$; water depth $=2207 \mathrm{~m}$ ). All data points from small populations (i.e., $<40$ individuals $/ 10$ $\mathrm{cm}^{3}$ ) have been excluded. Dotted lines join data points over sample gaps. Detailed palynological data from Core 76-029-033P are reported by de Vernal (1986) and de Vernal et al. (1987).

A very short stage 4 is present in Core 76-027-016P and at Site 645 (Fig. 7), with $\delta^{18} \mathrm{O}$ values again exceeding $+4 \%$, between about 8 and 9 mbsf.

The lower part of the isotopic record (down to $\sim 22 \mathrm{~m}$ in Cores $105-645 \mathrm{~B}-2 \mathrm{X}, 105-645 \mathrm{~F}-2 \mathrm{H}$, and $105-645 \mathrm{~F}-3 \mathrm{H})$ is more difficult to interpret. Relatively large-amplitude shifts in $\delta^{18} \mathrm{O}$ (Table 1) indicate episodes of glacial activity alternating with phases of meltwater discharge. Indeed, $\delta^{18} \mathrm{O}$ values exceeding $+4 \%$ point to ice growth over surrounding lands, as already proposed on theoretical grounds by Boulton (1979) and from field data on Baffin Island by Andrews et al. (1984). Owing to the few data points, the poor core recovery, and probable gaps in the record (Fig. 2), any substage delimitation for isotopic stage 5 is speculative. As will be shown later with the micropale- 


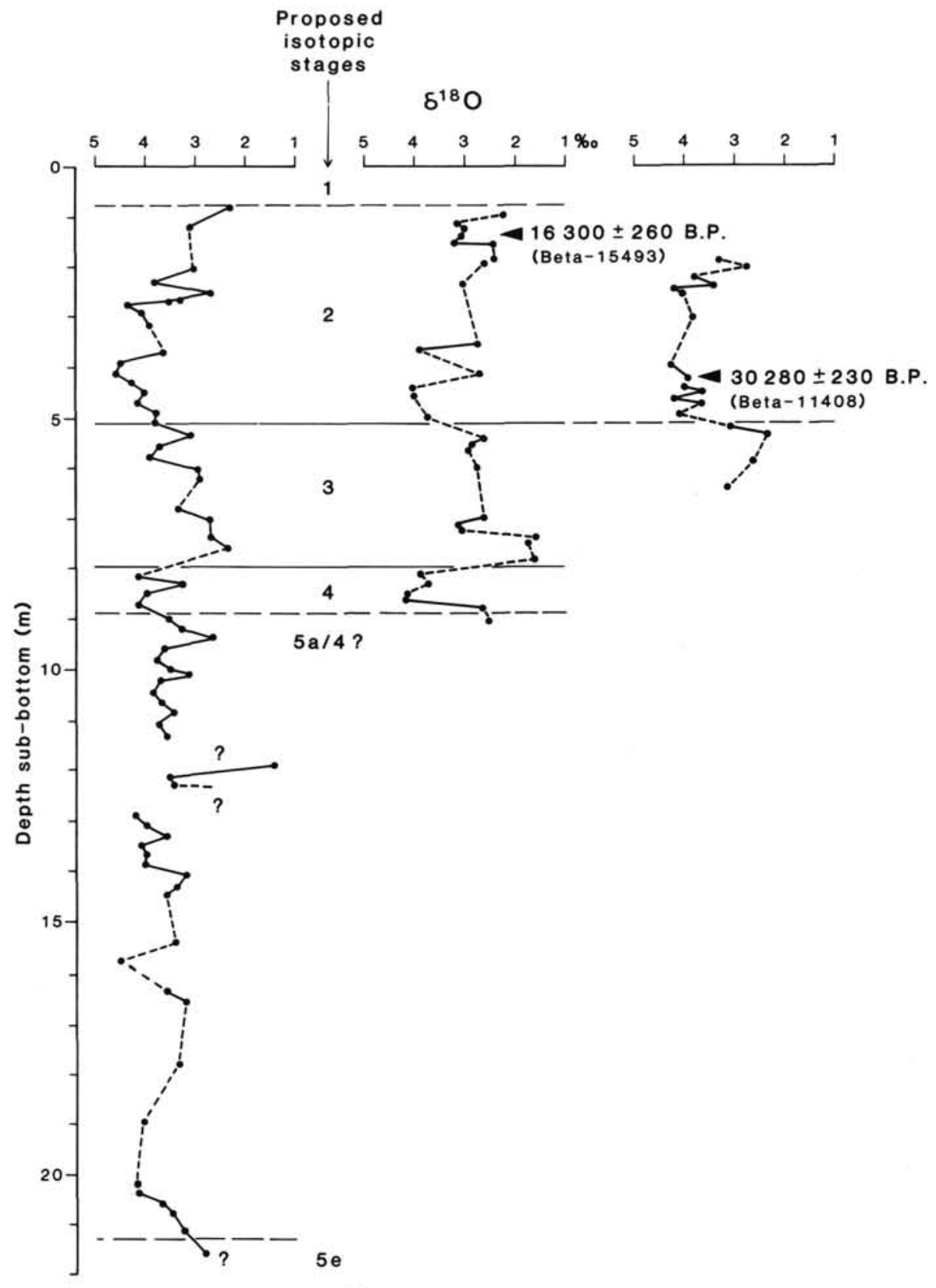

Figure 7. Proposed late Pleistocene ${ }^{18} \mathrm{O}$-stratigraphy in Baffin Bay.

ontological data, the lowermost part of the composite sequence (Section 105-645C-3H-5) probably belongs to substage 5e. If this interpretation were correct, stage 5 then might be characterized by high sedimentation rates of approximately $20 \mathrm{~cm} / \mathrm{k} . \mathrm{y}$.

\section{CARBON-ISOTOPE RECORD}

As a whole, $\delta^{13} \mathrm{C}$ values range from -0.5 to $+0.5 \%$ and do not show a clear inverse correlation with those of $\delta^{18} \mathrm{O}$, as also was observed by Labeyrie and Duplessy (1985) in the North Atlantic and the Labrador Sea. Figure 8A depicts the poor correlation between the two sets of data. Several variables are involved in the carbon budget at this latitude: (1) the $\mathrm{CO}_{2}$ exchange rate at the ocean/atmosphere interface in relation to the variable sea-ice cover, (2) the planktonic productivity (probably low here in general), (3) the oxidation of the organic matter during settling, and (4) the vertical displacement of living Neogloboquadrina pachyderma in the water column when surface water be- came more diluted. Because of all these variables, erratic trends would not be surprising in such an isolated basin. Actually, an inverse ${ }^{18} \mathrm{O}-{ }^{13} \mathrm{C}$ correlation occurs in parts of the sequence, notably in the lowermost section, which we believe represents isotopic substage 5e (Section 105-645C-3H-5; Fig. 4), whereas a positive correlation characterizes the sequence between 14 and 21 mbsf (Core 105-645F-3H; Fig. 8B). The latter interval is also the richest in reworked biogenic material, which possibly may explain the low $\delta^{13} \mathrm{C}$ values observed between 15 and 21 mbsf. These values fluctuate in parallel with those of $\delta^{18} \mathrm{O}$ and correspond to peaks in reworked palynomorphs that probably were transported into the basin by meltwater. Because Baffin Bay generally experienced low-planktonic carbon production, any influx of detrital organic carbon easily might cause an increased production of ${ }^{13} \mathrm{C}$-depleted $\mathrm{CO}_{2}$ following oxidation in the water column. During such episodes, the dilution of surface water might restrict foraminifers to their deepest habitats, i.e., a few 
A

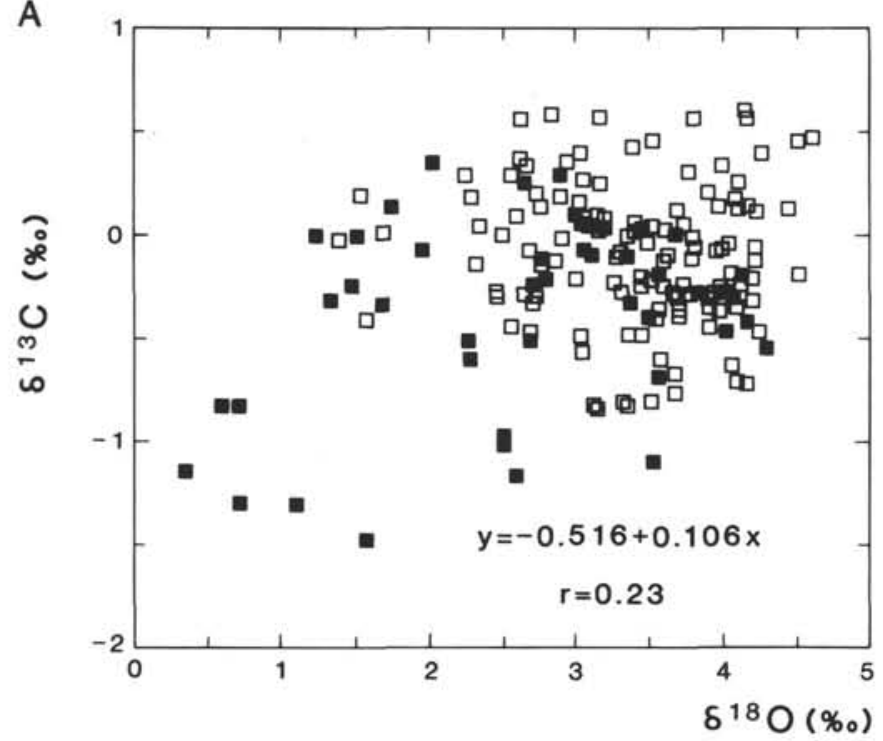

ㅁ $>40$ shells

- $<40$ shells

B

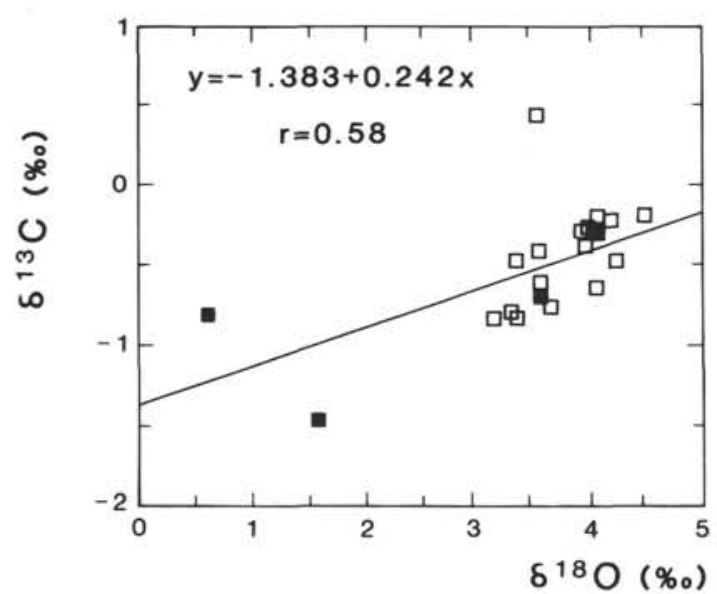

Figure 8 . The ${ }^{13} \mathrm{C}-{ }^{18} \mathrm{O}$ correlation in the studied cores. A. All samples were plotted; no trend was observed. B. Cores $105-645 \mathrm{~F}-2 \mathrm{H}$ and $105-$ $647 \mathrm{~F}-3 \mathrm{H}$ : correlated values were observed (see comments in text).

hundred meters for N. pachyderma (see Aksu, 1981), where oxidation would produce light inorganic carbon. In consequence, foraminifer shells then simultaneously might record lower ${ }^{18} \mathrm{O} /$ ${ }^{16} \mathrm{O}$ and ${ }^{13} \mathrm{C} /{ }^{12} \mathrm{C}$ ratios.

\section{ORGANIC CARBON AND NITROGEN RECORDS}

The upper $22 \mathrm{~m}$ of sediments at Site 645 is characterized by generally low concentrations in organic carbon $(1.0 \%)$ and total nitrogen $(<0.1 \%$; Fig. 9; Table 2$)$. This suggests a generally low biogenic productivity (Müller and Suess, 1979). The $\delta^{13} \mathrm{C}$ and $\delta^{15} \mathrm{~N}$ curves (Fig. 9) show sharp large-amplitude fluctuations between -28 and $-21 \%$ and between 3 and $11 \%$, respectively. Stable carbon-isotope fluctuations are a consequence of variable influxes of terrestrial and marine organic matter. In Baffin Bay, the terrestrial influx seems predominant in view of the generally low $\delta^{13} \mathrm{C}$ values (Nissembaum, 1974). This and the low organic carbon content in the sediments strongly suggest a poor marine biogenic productivity.

The lower part of the record, between approximately 15 and 20 mbsf, is marked by slightly increased nitrogen and carbon concentrations, low ${ }^{13} \mathrm{C} /{ }^{12} \mathrm{C}$ ratios in organic matter, and simi- larly low $\delta^{13} \mathrm{C}$ values in foraminifers. This change may be attributed to a significant influx of terrestrial organic matter, which is also supported by the abundance of reworked pre-Quaternary palynomorphs in the 14 - to $21-\mathrm{m}$ interval (see following).

\section{PALYNOLOGIC RESULTS}

Pleistocene sediments from Baffin Bay usually contain very few dinoflagellate cysts (Mudie and Short, 1985; de Vernal, 1986; de Vernal et al., 1987). In Pleistocene samples from Site 645 and Core 85-027-016, the calculated concentrations are generally lower than 100 cysts $/ \mathrm{cm}^{3}$ (Figs. 5, 10, 11, and 12). Extrapolated from the mean sedimentation rates (about $10-20 \mathrm{~cm}$ / k.y.), the corresponding influxes $\left(<2 \mathrm{cysts} / \mathrm{cm}^{2} / \mathrm{yr}\right)$ indicate low local dinoflagellate productivity. However, a few samples yielded more abundant dinocysts, which indicates brief but significant episodes of phytoplanktonic productivity. In particular, the surface samples (Fig. 11: Sample 105-645A-1H-1, 0-2 cm; Fig. 12: Core 85-027-016TWC, 5-7 cm) and the lower two samples of the composite sequence (Fig. 11: 105-645C-3H-5, 92-94 cm; $105-645 \mathrm{C}-3 \mathrm{H}, \mathrm{CC})$ are characterized by dinocyst assemblages dominated by subarctic taxa, such as Operculodinium centrocarpum and Spiniferites elongatus (Harland et al., 1980; Harland, 1983; Mudie and Short, 1985). These assemblages may be associated with subpolar conditions in surface-water masses similar to those prevailing today and doubtless are related to the penetration of subarctic water through the Davis Strait. Otherwise, the high dinocyst concentrations recorded throughout the recovered sequence correspond to peaks of Multispinula minuta and Brigantedinium simplex, which suggest arctic conditions and a low salinity in the surface waters (about $30 \%$; Mudie and Short, 1985).

The Quaternary pollen and spore concentrations are generally very low in the Pleistocene sediments from Baffin Bay (Figs. 5 and 10; Table. 3; see also Mudie and Short, 1985; de Vernal et al., 1987). Consequently, detailed interpretation was not possible. Nevertheless, the restricted pollen influx indicates a reduced vegetation cover on surrounding lands. The dominance of Pinus (Table 3), characterized by a morphology favorable to atmospheric transportation over long distances (e.g., Mudie, 1982; Heusser, 1983), reveals influxes from southern areas and also points to the low productivity of the regional vegetation. One exception should be mentioned: at the base of the high-resolution sequence, the two lowermost samples (105-645C-3H-5, 92$94 \mathrm{~cm}$, and $105-645 \mathrm{C}-3 \mathrm{H}, \mathrm{CC})$ are characterized by relatively high concentrations of Quaternary terrestrial palynomorphs $\left(>500 / \mathrm{cm}^{3}\right)$. Furthermore, the pollen and spore spectra, although still dominated by Pinus, reveal significant proportions of other taxa, such as Betula $(\sim 9 \%)$, Alnus crispa $(\sim 5 \%)$, Sphagnum $(13 \%-20 \%)$, and herbs $(6 \%-8 \%)$. This assemblage suggests relatively high regional productivity and a shrub-tundra vegetational cover, probably over Baffin Island and possibly over Greenland. The higher Picea percentages $(13 \%-14 \%)$ also point to a northward shift of the boreal-forest limit (Mudie, 1982; de Vernal, 1986). These arguments support assigning the lowest part of the high-resolution record to an interglacial interval.

One of the main features in the palynostratigraphy of the upper $22 \mathrm{~m}$ of sediments is the abundance of reworked pre-Quaternary palynomorphs originating from sedimentary formations that outcrop around Baffin Bay (Fig. 10). Most of the reworked palynomorphs are terrestrial and consist of trilete spores, bisaccates, and angiosperm pollen grains (Table 3 ). These palynomorphs are characterized by a high degree of compression and a brownish color. The diagenetic alteration of the sporopollenin also can be distinguished by fluorescent microscopy. Taxa identification is not easy because of the poor preservation of these palynomorphs, particularly for the trilete spores and bisaccates. Nevertheless, Cicatricosisporites, Appendicisporites, and Camar- 


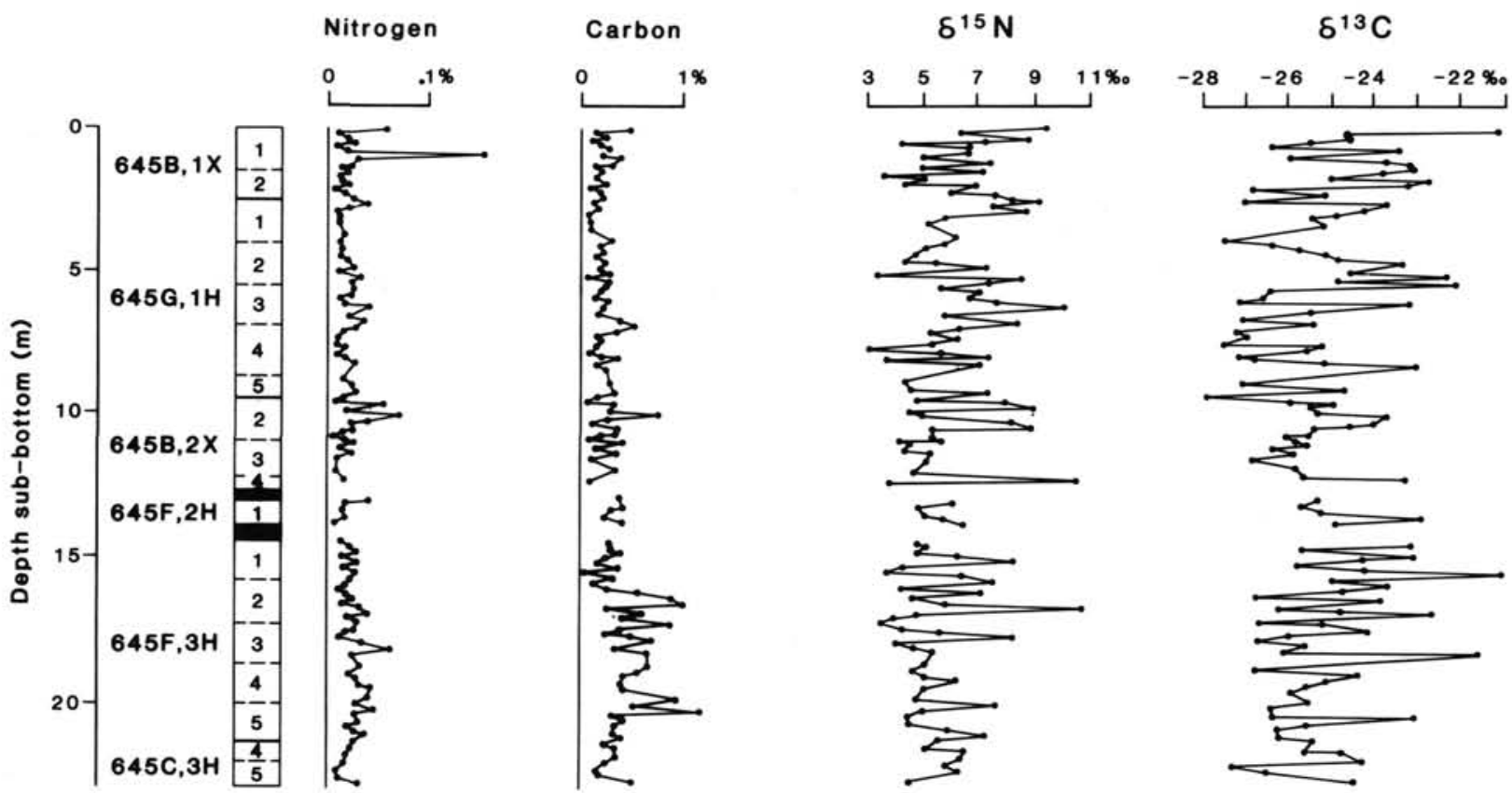

Figure 9. Organic-carbon and nitrogen concentrations and isotopic compositions in the composite sequence of Site 645.

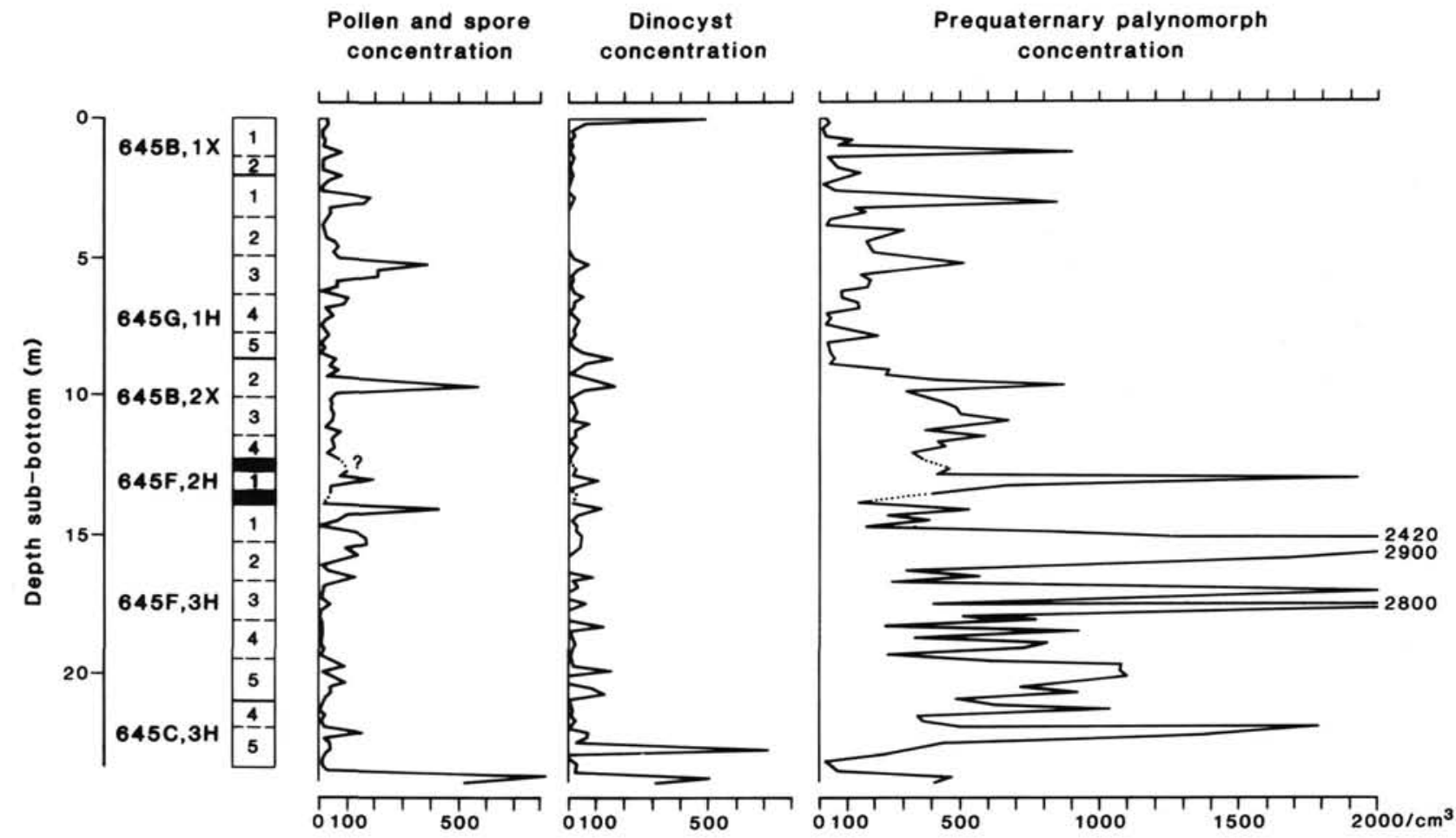

Figure 10. Palynomorph concentrations in the composite sequence of Site 645.

ozonosporites were identified in many samples. Most reworked angiosperm pollen grains belong to the genus Triporopollenites. A few reworked dinocysts and acritarchs are generally present. Within the pre-Quaternary marine palynomorph assemblages, Veryhachium sp., Wetzeliella articulata, Chatangiella granulifera, and Deflandrea spp. also were frequently observed. The exact age of the reworked palynoflora is difficult to determine since most assemblages show a mixture of palynoflora from the
Paleozoic to Paleogene. However, the common angiosperm pollen grains and the nature of the reworked dinocysts suggest a noticeable erosion of Cretaceous to Paleogene strata.

As mentioned earlier, these reworked palynomorphs are particularly abundant in the lower part of the sequence (Cores 105645B-2X, 105-645F-2H, and 105-645F-3H; Fig. 10). They are probably related to glacial erosion, transport, and outwash deposition from adjacent lands and especially from the Arctic Ar- 


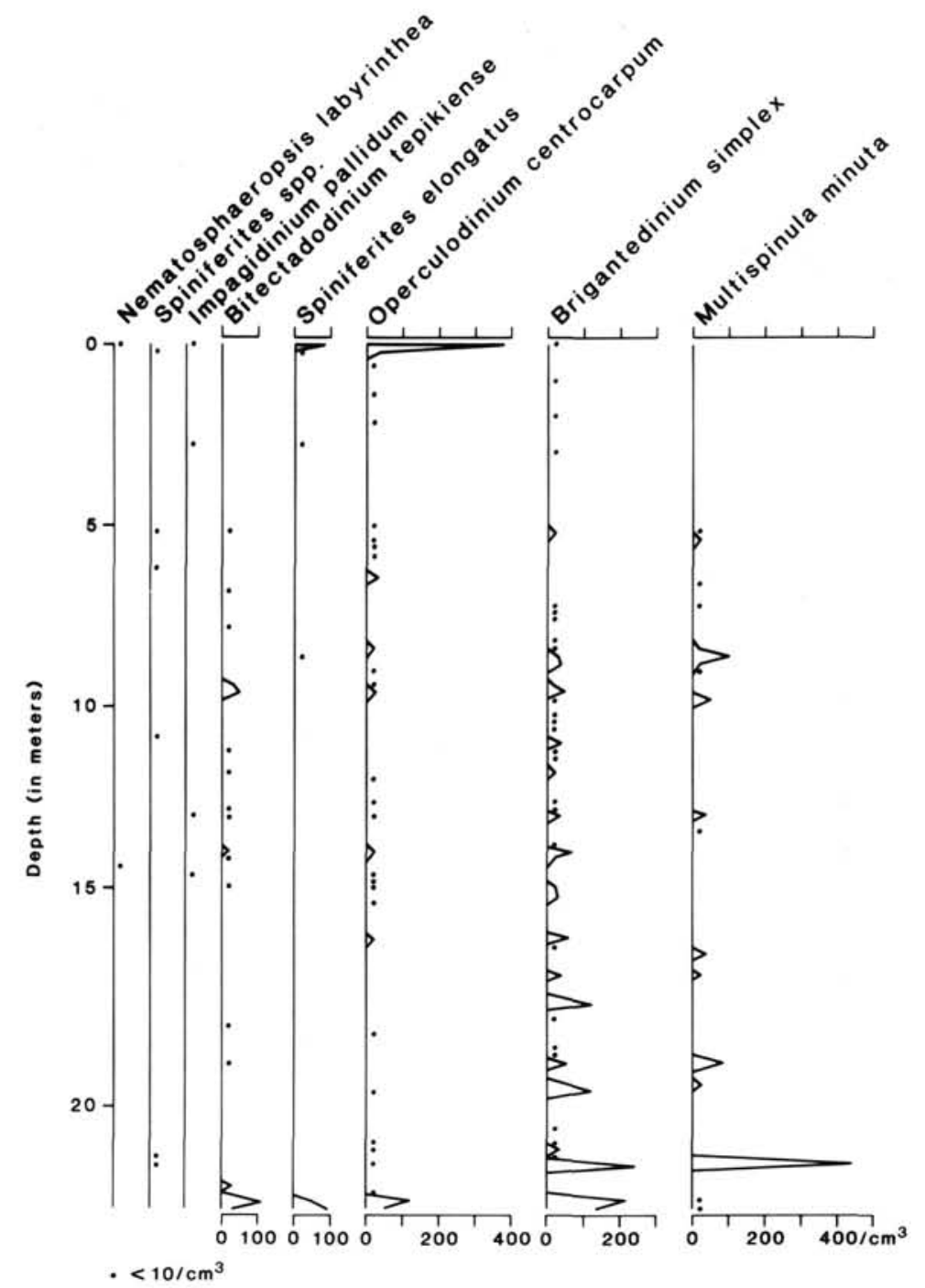

Figure 11. Quaternary dinocyst concentrations at Site 645 .

chipelago, where the original sedimentary units outcrop. The most severe glacial activity probably occurred during this interval, which is attributed to isotopic stage 5 on the basis of the ${ }^{18} \mathrm{O}$ stratigraphy. Continental stratigraphies in the Canadian Arctic also show major glaciation at the beginning of the late Pleistocene, probably during isotopic stage 5 (e.g., Andrews et al., 1984,; Andrews and Miller, 1984; Klassen, 1985). Thus, direct land-sea correlations appear possible.

\section{PLANKTONIC FORAMINIFER RECORD}

The surface sample at Site 645 and those from several (about 50) piston cores collected throughout Baffin Bay (Aksu, 1981, 1983) were barren of planktonic and calcareous benthic foraminifers. However, plankton tows from the water column in Baffin Bay contain planktonic foraminifer fauna dominated by Neogloboquadrina pachyderma (sinistral) and characterized by $7 \%-10 \%$ subpolar species, including $N$. pachyderma (dextral), G. bulloides, and G. quinqueloba (Stehman and Gregory, 1973; Vilks, 1974). In the upper $\sim 22 \mathrm{~m}$ of sediments at Site 645 , the total abundance of planktonic foraminifers is generally low, but large fluctuations from 0 to 10,000 specimens/g were observed (Fig. 13). The planktonic foraminifer assemblage consists predominantly of the polar species, Neogloboquadrina pachyderma (sinistral), and varying but small percentages of the subpolar species, N. pachyderma (dextral), Globigerina bulloides, Globigerina quinqueloba, Globigerinitida uvula, and Globigerinitida glutinata.

The fluctuations in total foraminifer abundance may result from several parameters: (1) cyclical changes in the preservation of calcium carbonate debris on the seafloor (Aksu, 1983), (2) changes in sedimentation rate and processes, or (3) changes in the oceanographic conditions and circulation patterns accompanied by changes in biological productivity. Semiquantitative assessment of calcium carbonate dissolution is based on (1) the ratio of benthic to planktonic foraminifers (B/P; see Thunell, 1976; Aksu, 1983), (2) the degree of foraminifer test fragmentation (Thunell, 1976), (3) the percentage of dissolution susceptible vs. dissolution resistant planktonic foraminifers (Ruddiman and Heezen, 1967), and (4) the plankton tow data compared with the fauna in surface sediments (Vilks, 1974). One or possibly two intervals with calcium carbonate dissolution were identified in the high-resolution record of the upper $\sim 22 \mathrm{~m}$ at Site 645 . In surface sediments (about $0-20 \mathrm{~cm}$ ), the absence of planktonic foraminifers, despite their presence in plankton tows (Vilks, 1974), as well as the downslope trend of decreasing calcareous benthic foraminifers (Aksu, 1983), the high $\mathrm{B} / \mathrm{P}$ ratio 


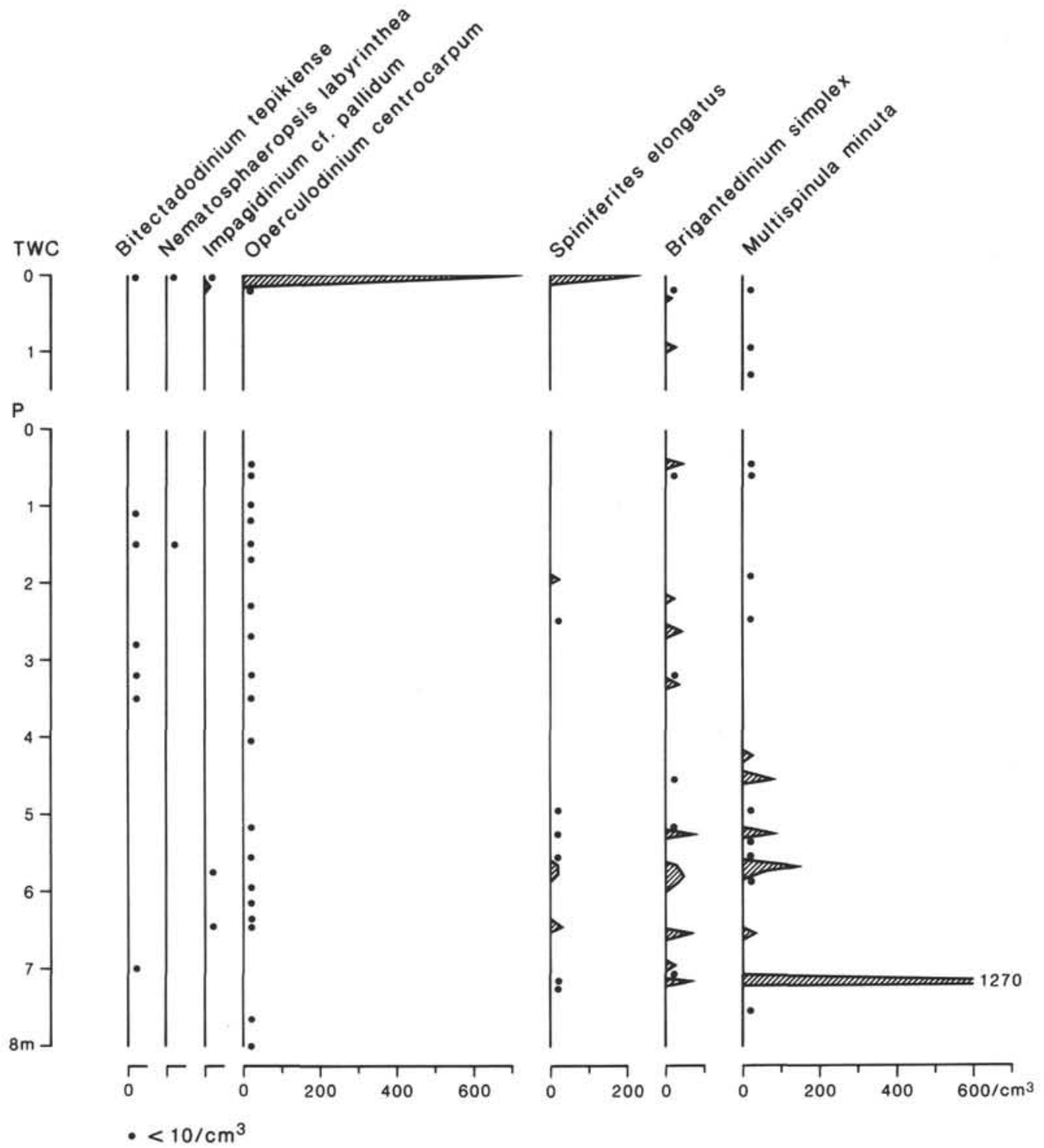

Figure 12. Quaternary dinocyst concentrations in Core 85-027-016.

and the foraminifer test fragmentation clearly indicate calcium carbonate dissolution in Baffin Bay during the Holocene. Between approximately 15 and $19 \mathrm{~m}$ (Sections $105-645 \mathrm{~F}-3 \mathrm{H}-2$ to 105-645F-3H-4; Fig. 13), variable but high $\mathrm{B} / \mathrm{P}$ ratios might possibly be interpreted as a consequence of calcium carbonate dissolution, although test fragmentation is moderate $(0 \%-12 \%)$ and does not really support this hypothesis. Apart from these two intervals, the data suggest minimal dissolution of the foraminifers. Therefore, the relatively low planktonic foraminifer concentrations in the upper $22 \mathrm{~m}$ suggest a generally low zooplanktonic production, in particular during the two intervals that correspond to isotopic stages 3 and 5 . In most samples, the subpolar species constitute less than $3 \%$ of the faunal assemblages, which indicates a predominance of polar-water masses that were probably colder than those of today.

\section{CONCLUSIONS}

As shown by biostratigraphic and geochemical records, Baffin Bay was characterized by low planktonic productivity throughout most of the late Pleistocene. This low productivity probably was caused by harsh arctic conditions and extensive sea-ice cover or by low salinities in the surface-water masses.
Despite the relatively large amplitude shifts in oxygen-isotope ratios, an ${ }^{18} \mathrm{O}$ stratigraphy spanning the last five isotopic stages can be reasonably proposed. However, isotopic particularities in the lower part of the record and poor core recovery prevent a firm age assignment for the base of the studied sedimentary sequence. Nevertheless, the lowest section probably corresponds to isotopic substage $5 \mathrm{e}$, according to palynological data that indicate interglacial conditions. The proposed stratigraphy and a few AMS ${ }^{14} \mathrm{C}$ dates on handpicked foraminifers allowed us to calculate high but variable sedimentation rates that range between about 10 to $20 \mathrm{~cm} / \mathrm{k}$.y. during the late Pleistocene.

The concurrence of light ${ }^{18} \mathrm{O}$-peaks and low-salinity dinocyst assemblages points to episodic influxes of large amounts of meltwater in Baffin Bay, notably during isotopic stages 3 and 5 , in response to adjacent ice-sheet fluctuations. The most obvious evidence of an intense glacial activity over the surrounding land can be found in the high concentration of reworked pre-Quaternary palynomorphs in the lower one-half of the sequence, which we assume belongs to isotopic stage 5. The Baffin Bay depositional history thus is related to the glacial history of borderlands, and direct correlations with Baffin Island and/or Greenland stratigraphies can be established. 


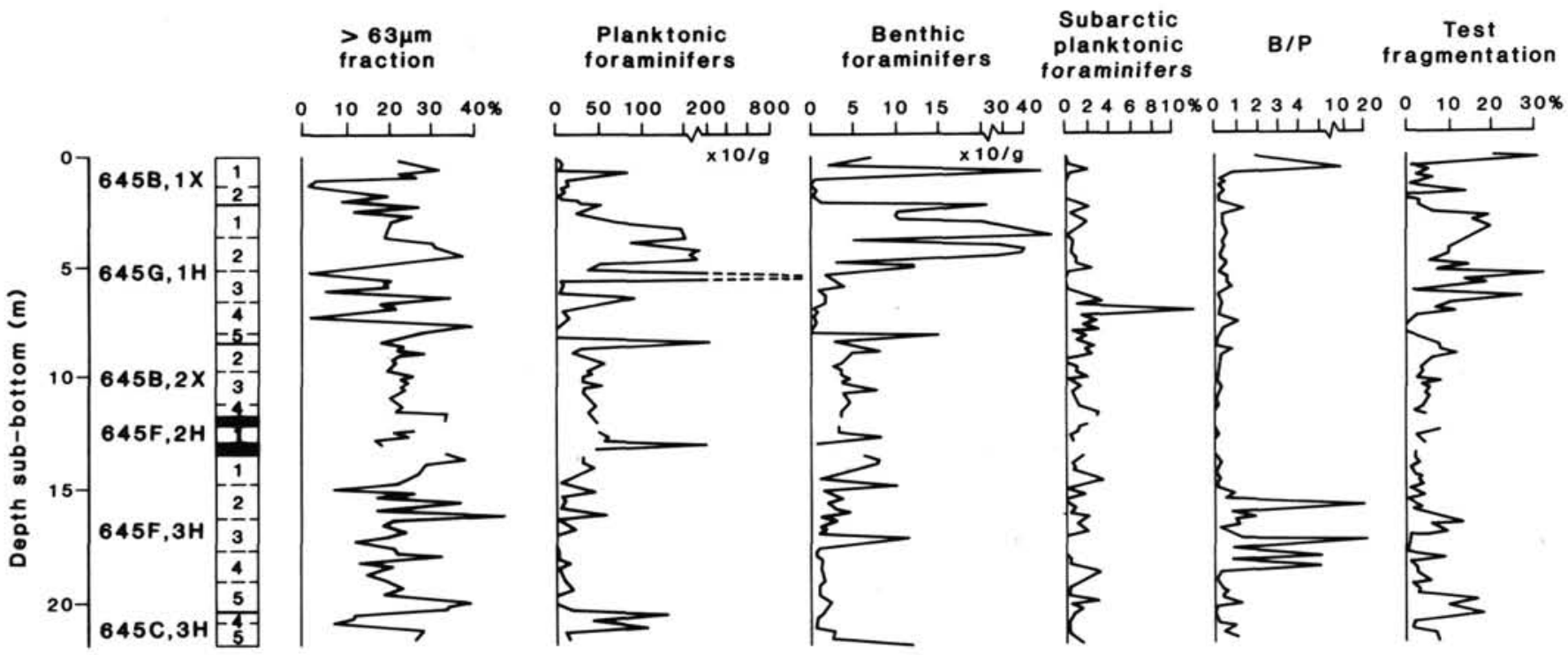

Figure 13. Sand concentrations and summarized foraminifer stratigraphy in the composite sequence of Site 645.

\section{ACKNOWLEDGMENTS}

Technical support was provided by O. Carro, C. Goyette, and C. Guilmette; analyses by S. Hookey and K. Pauley; and drawings by M. Laithier. We especially thank M. Arthur and S. Srivastava, Co-Chief Scientists of Leg 105, and B. Maclean, Chief Scientist of the Hudson during the Site 645 survey, who kindly contributed time to piston coring of surficial sediments, which enabled us to obtain a high-resolution study of the last glacial cycle. Critical reading by J. Andrews (INSTAAR-Colorado), S. Srivastava (AGC-Canada), and an anonymous reviewer helped to clarify several points. EMR Canada support to ODP, financial assistance from NSERC-Canada (Grants A-9156 and CSP-Leg 105), and FCAR Funds of Québec (Grant EQ-492) were essential.

\section{REFERENCES}

Aksu, A. E., 1981. Late Quaternary stratigraphy, paleoenvironments and sedimentation history of Baffin Bay and Davis Strait [Ph.D. dissert.]. Dalhousie University, Nova Scotia.

1983. Holocene and Pleistocene dissolution cycles in deepsea cores of Baffin Bay and Davis Strait: paleoceanographic implications. Mar. Geol., 53:331-348.

1984. Subaqueous debris flow deposits in Baffin Bay. GeoMar. Lett., 4:83-90.

1985. Climatic and oceanographic changes over the past 400,000 years: evidence from deep-sea cores on Baffin Bay and Davis Strait. In Andrews, J. T. (Ed.), Quaternary Environments, Eastern Canadian Arctic, Baffin Bay and Western Greenland: London (Allen and Unwin), 181-209.

Aksu, A. E., and Piper, D.J.W., 1979. Baffin Bay in the past 100,000 years. Geology, 7:245-248.

1987. Late Quaternary sedimentation in Baffin Bay. Can. J. Earth Sci., 24:1833-1846.

Andrews, J. T., and Miller, G. H., 1984. Quaternary glacial and nonglacial correlations for Eastern Canadian Arctic. In Fulton, R. J. (Ed.), Quaternary Stratigraphy of Canada. Geol. Surv. Can., Pap. 84-10: 101-116.

Andrews, J. T., Miller, G. H., Vincent, J. S., and Shilts, W. W., 1984. Quaternary correlations in Arctic Canada. In Fulton, R. J. (Ed.), Quaternary Stratigraphy of Canada. Geol. Surv. Can. Pap., 84-10: 127-134.

Bédard, P., Hillaire-Marcel, C., and Pagé, P., 1981. ${ }^{18} \mathrm{O}$-modelling of freshwater inputs in Baffin Bay and Canadian Arctic coastal waters. Nature, 293:287-289.

Boulton, G. S., 1979. A model of Weichselian glacier variation in the North Atlantic region. Boreas, 8:373-395.

Broecker, W. S., 1975. Floating glacial ice caps in the Arctic Ocean. Science, 188:1116-1118.
Dansgaard, W., Johnsen, S. J., Clausen, H. B., and Langway, C. C., Jr., 1971. Climatic record revealed by the Camp-Century ice core. In Turekian, K. E., (Ed.), Late Cenozoic Glacial Ages: New Haven (Yale University Press), 37-56.

de Vernal, A., 1986. Analyses palynologiques et isotopiques de sédiments de la mer du Labrador et de la baie de Baffin. Eléments d'une climatostratigraphie du Pléistocène supérieur dans l'est du Canada [Ph.D. dissert.]. Université de Montréal, Canada.

de Vernal, A., and Hillaire-Marcel, 1987. Marginal paleoenvironments of the eastern Laurentide Ice-Sheet and timing of the last ice maximum and retreat. Géogr. Phys. Quat., 41:265-277.

de Vernal, A., Hillaire-Marcel, C., Aksu, A. E., and Mudie, P. J., 1987. Palynostratigraphy and chronostratigraphy of Baffin Bay deep sea cores: climatostratigraphic implications. Palaeogeogr., Palaeoclimatol., Palaeoecol., 61:97-105.

Duplessy, J.-C., 1978. Isotope studies. In Gribbin, J. (Ed.), Climatic Change: London (Cambridge University Press), 46-67.

Fillon, R. H., 1985. Northwest Labrador Sea stratigraphy, sand input and paleoceanography during the last 160,000 years. In Andrews, J. T. (Ed.), Quaternary Environments, Eastern Canadian Arctic, Baffin Bay and Western Greenland: London (Allen and Unwin), 210247.

Fillon, R. H., and Duplessy, J.-C., 1980. Labrador Sea bio-, tephro-, oxygen isotopic stratigraphy and late Quaternary paleoceanographic trends. Can. J. Earth Sci., 17:831-854.

Harland, R., 1983. Distribution maps of recent dinoflagellate cysts in bottom sediments from the North-Atlantic Ocean and adjacent seas. Paleontology, 26:321-387.

Harland, R., Reid, P. C., Dobell, P., and Norris, G., 1980. Recent and subrecent dinoflagellate cysts from the Beaufort Sea, Canadian Arctic. Grana, 19:211-225.

Heuser, L. E., 1983. Pollen distribution in the bottom sediments of the western North-Atlantic Ocean. Mar. Micropaleontol., 8:77-88.

Klassen, R. A., 1985. An outline of glacial history of Bylot Island, District of Flanklin, N.W.T. In Andrews, J. T. (Ed.), Quaternary Environments, Eastern Canadian Arctic, Baffin Bay and Western Greenland: London (Allen and Unwin), 428-460.

Labeyrie, L. D., and Duplessy, J.-C., 1985. Change in the oceanic ${ }^{13} \mathrm{C} /$ ${ }^{12} \mathrm{C}$ ratio during the last 140,000 years: high-latitude surface water records. Palaeogeogr., Palaeoclimatol., Palaeoecol., 50:217-240.

Matthews, J., 1969. The assessment of a method for the determination of absolute pollen frequencies. New Phytol., 68:161-166.

Mudie, P. J., 1982. Pollen distribution in recent marine sediments, Eastern Canada. Can. J. Earth Sci., 19:729-747.

Mudie, P. J., and Aksu, A. E., 1984. Paleoclimate of Baffin Bay from 300,000 year record of foraminifera, dinoflagellate and pollen. $\mathrm{Na}$ ture, 312:630-634. 
Mudie, P. J., and Short, S. K., 1985. Marine palynology of Baffin Bay. In Andrews, J. T. (Ed.), Quaternary Environments, Eastern Canadian Arctic, Baffin Bay and Western Greenland: London (Allen and Unwin), 263-307.

Muench, R. D., 1971. The physical oceanography of the northern Baffin Bay region. Sci. Rept. Arctic Inst. N. Am., 1.

Nissenbaum, A., 1974. The organic geochemistry of marine and terrestrial humic substances: implication of carbon and hydrogen isotope studies. In Tissot, B., and Bienner, F. (Eds.), Advances in Organic Geochemistry: Amsterdam (Elsevier), 39-52.

Ruddiman, W. F., and Heezen, B. C., 1967. Differential solution of planktonic foraminifera. Deep-Sea Res., 14:801-808.

Shackleton, N. J., and Opdyke, N. D., 1973. Oxygen isotope and paleomagnetic stratigraphy of Equatorial Pacific core V-28-238. Oxygen isotope temperatures and ice volumes on a $10^{5}$ year scale. Quat. Res., 3:39-55.

Shackleton, N. J., Imbrie, J., and Hall, M. A., 1983. Oxygen and carbon isotope record of East Pacific core V19-30: implications for the formation of deep water in the late Pleistocene North Atlantic. Earth Planet. Sci. Lett., 65:233-244.

Srivastava, S. P., Arthur, M., et al., 1987. Proc. ODP, Init. Repts., 105: College Station, TX (Ocean Drilling Program).
Stehman, C. F., and Gregory, M., 1973. A preliminary account of benthic and planktonic foraminifera in Baffin Bay, Davis Strait and the Labrador Sea. Geol. Surv. Can., Pap. 71-23:499-507.

Tan, F. C., and Strain, P. M., 1980. The distribution of sea ice meltwater in the Eastern Canadian Arctic. J. Geophys. Res., 85:19251932.

Thouveny, N., in press. High-resolution study of late Pleistocene paleomagnetic variations recorded in a sedimentary sequence from Baffin Bay. Can. J. Earth Sci.

Thunell, R. C., 1976. Calcium carbonate dissolution in late Quaternary deep-sea sediments, Western Gulf of Mexico. Quat. Res., 6:281297.

Vilks, G., 1974. The distribution of planktonic foraminifera in sediments and water of the Northwest Passage and northern Baffin Bay; a tool for paleoceanographic synthesis. Geol. Surv. Can. Pap., 7430:109-121.

Date of initial receipt: 30 June 1987

Date of acceptance: 7 June 1988

Ms 105B-138 